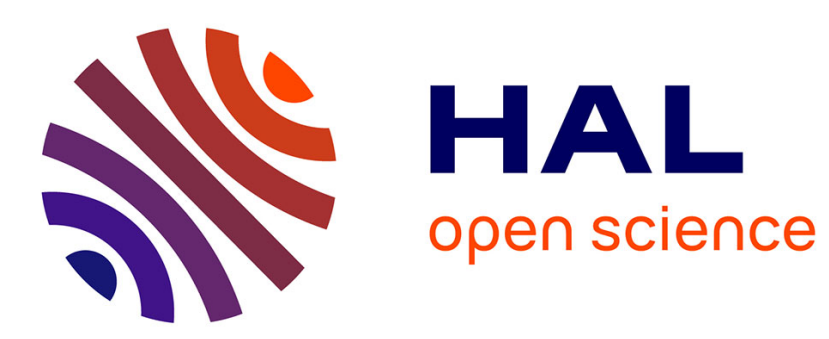

\title{
Effect of shear on a lyotropic lamellar phase
}

Olivier Diat, Didier Roux, Frédéric Nallet

\section{To cite this version:}

Olivier Diat, Didier Roux, Frédéric Nallet. Effect of shear on a lyotropic lamellar phase. Journal de Physique II, 1993, 3 (9), pp.1427-1452. 10.1051/jp2:1993211 . jpa-00247917

\section{HAL Id: jpa-00247917 https://hal.science/jpa-00247917}

Submitted on 1 Jan 1993

HAL is a multi-disciplinary open access archive for the deposit and dissemination of scientific research documents, whether they are published or not. The documents may come from teaching and research institutions in France or abroad, or from public or private research centers.
L'archive ouverte pluridisciplinaire HAL, est destinée au dépôt et à la diffusion de documents scientifiques de niveau recherche, publiés ou non, émanant des établissements d'enseignement et de recherche français ou étrangers, des laboratoires publics ou privés. 
Classification

Physics Abstracts

$61.30-82.70-46.60$

\title{
Effect of shear on a lyotropic lamellar phase
}

\author{
Olivier Diat $\left(^{*}\right)$, Didier Roux and Frédéric Nallet \\ Centre de recherche Paul-Pascal, CNRS, avenue du Docteur Schweitzer, 33600 Pessac, France
}

(Received 17 March 1993, accepted 18 May 1993)

\begin{abstract}
We present a series of experiments, using different techniques such as light scattering, conoscopy, neutron scattering and microscopic observations, to determine the orientation of lyotropic lamellar phases under shear. Three states of orientation are observed, depending upon both the shear rate and the inter-membrane separation. These steady states are separated by dynamic transitions. Among the states described, we focus our attention on a state made of monodisperse close-packed multilayered vesicles whose size is precisely fixed by the shear rate.
\end{abstract}

\section{Introduction.}

Surfactants in solution are known to lead, in concentrated regimes (surfactant ratio $>30 \%$ ), to lyotropic liquid crystals [1]. In certain cases, such long-range-ordered phases may persist over a very large range of dilution. This is the case for dilute lamellar phases (lyotropic smectics A) which have been extensively studied [2-4]. Indeed, the ability of preparing phases made of membranes with varying continuously the intermembrane separation has allowed quantitative measurements of membrane properties [3, 4]. Owing to its liquid crystalline nature, the lyotropic lamellar phase possesses static and dynamic properties associated to both the crystalline organization (long-range translational order, elasticity) and the liquid one (viscosity, concentration fluctuations...). The measurements of macroscopic properties such as elastic constants, have allowed researchers to obtain information on the interactions and flexibility of membranes constituting the smectic phase. For example, it has been possible to demonstrate that dilute lamellar phases are stabilized either by weakly-screened electrostatic repulsions [5] or by undulation forces [3-8]. One can now consider that a large part of the equilibrium behaviour of these phases has been understood both from a microscopic viewpoint (stack of interacting membranes) and from a macroscopic one (two-component smectic $\mathrm{A}$ elasticity). Much less is known concerning their out-of-equilibrium properties.

The effect of shear on the structure of a phase is assumed to be a perturbation of or a change in its spatial organization. However, one has to distinguish between long-range-ordered

(*) Present address : Installation européenne de rayonnement synchrotron, ESRF, B.P. 220, avenue des Martyrs, 38043 Grenoble Cedex, France. 
phases, where even a small shear can produce large effects such as shear melting [9] and liquid phases where expectations to modify the short-range order arises only when the shear rate is high enough to be of the order of a characteristic frequency of the associated fluctuations. The ratio between shear rate and this characteristic frequency is called the Deborah number $(D e)$. For simple liquids the relevant characteristic frequency is in the range $10^{10}-10^{12} \mathrm{~s}^{-1}$ which makes experimentally accessible Deborah numbers very small. In systems where large characteristic distances (and consequently small characteristic frequencies) are found, the effect of shear can be experimentally studied. This is the case for instance in colloidal systems [10], critical fluids [11] or liquid crystalline phases close to the smectic-nematic phase transition [12].

With dilute lamellar phases having characteristic distances of several hundred Angströms, one expects several interesting features to take place. First, in regular liquid crystals, one not only expects an orientation of the phase under flow [13], but also a change in the structure, or at least of the smectic properties : Deborah numbers of the order of 1 are reached for reasonable shear rates $\left(10^{3} / 10^{4} \mathrm{~s}^{-1}\right)$.

In this study we report a series of experiments characterizing some effects of the shear on dilute lamellar phases. We mainly focus on the orientation of the system under shear. Using visual observations, scattering techniques and birefringence measurements an « orientation diagram » is built that characterizes the various steady-state orientations as a function of shear rate and dilution.

The system studied is a quaternary mixture of SDS (sodium dodecyl-sulphate), pentanol, water and dodecane. The dilution consists in adding dodecane + pentanol ( $9 \%$ in weight) to an initially concentrated lamellar phase water over surfactant mass ratio : 1.55 ; dodecane (resp. pentanol) weight fraction : $31 \%$ (resp. $18 \%$ ) $\}$. The phase diagram (see Fig. 1) is given in previous papers [2]. The lamellar phase of this system is stabilized by undulation forces [5]. Upon dilution the repeating distance varies from $40 \AA$ to $400 \AA$.

We briefly describe the cells used for birefringent and neutron scattering measurements and give the orientation diagram, as it results from our observations. Finally, we describe the properties of two of the steady orientation states found.

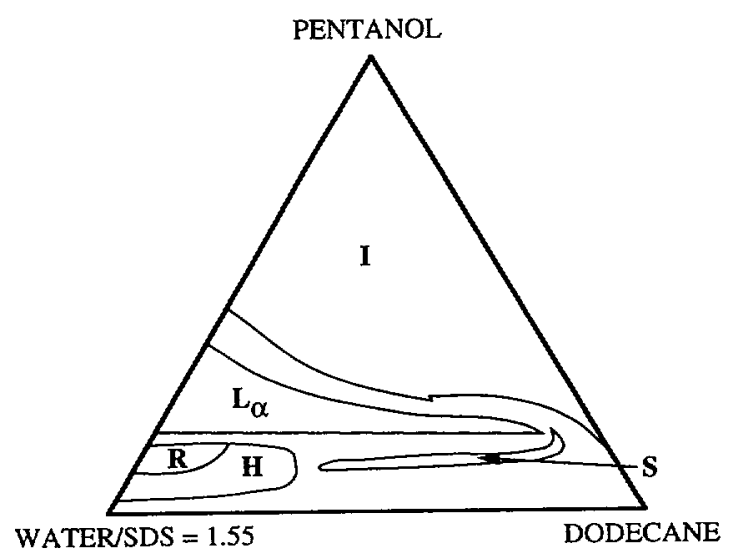

Fig. 1. - Cut of the phase diagram of the quaternary system water, pentanol, dodecane and sodium dodecylsulphate. The water : surfactant ratio is fixed to 1.55 . The symbol $\mathrm{L}_{\alpha}$ denotes the location of the lyotropic lamellar phase. 


\section{Shear cells.}

Couette cells made up of cylinders and ancillary windows of glass were used for direct observations and optical experiments. For neutron scattering experiments, Couette cells made of quartz instead of glass were employed. The two concentric cylinders have radii of about $30 \mathrm{~mm}$ and heights of $60 \mathrm{~mm}$. The gap between the cylinders may be varied (changing the inner cylinder), by $0.5,1$ or $2 \mathrm{~mm}$. This Couette geometry is chosen because it yields a flow with (quasi) constant shear rate in the laminar regime when the gap is filled with a simple Newtonian fluid. Moreover the inner cylinder (stator) is fixed while the outer one (rotor) rotates, in order to avoid the Taylor instability. Depending upon gap and rotor angular velocity, the shear rate $\dot{\gamma}$ varies from 0 to $8000 \mathrm{~s}^{-1}(\dot{\gamma}=R \omega / e, e$ is the gap size, $\omega$ the angular velocity and $R$ the (inner) radius of the rotor). When the gap is filled with liquid crystalline samples, the flow is probably more complex than the simple, laminar flow one encounters with regular fluids. We nevertheless kept the name «shear rate » and notation $\dot{\gamma}$ for $R \omega / e$. Because our samples are sensitive to evaporation and temperature variations, the Couette cylinders are contained inside a thermostated and sealed oven (see Fig. 2). Four windows on the oven allow visual observations and neutron or light irradiation. The neutron scattering cell has been made in collaboration with laboratoire Léon-Brillouin (CEA/CNRS, Saclay, France), where the neutron scattering experiments have been performed.

A plate/plate cell, made of a rotating glass plate situated at a given distance from a fixed one (schematically drawn in Fig. 3) has also been used. Such a cell can fit in an optical microscope to allow direct space observations. The shear rate is no longer constant but varies continuously with the distance from the rotation axis. However, for thicknesses of 0.1 to $1 \mathrm{~mm}$ there is no appreciable variation of the shear rate over the microscope field (about $1 \mathrm{~mm}^{2}$ ).

Since we are interested in describing the orientation of a lamellar phase in a Couette cell, we define the eigen directions as follows. We refer to the cylindrical coordinate axes of the Couette cell X, Y and Z; (see Fig. 2) and to the smectic axis with unit vector $\mathbf{n}$ normal to the layers. We define $X$ to be the (tangential) velocity axis, $Y$ the (radial) velocity gradient axis and $\mathrm{Z}$ the (neutral) rotation axis.

\section{Visual observations.}

Several samples have been studied along a dilution line. They correspond to different oil concentrations and consequently have different $d$-spacings. For each sample visual observations between crossed polarizers as a function of the shear rate are made either directly by eye on the Couette cell, or under the microscope using the plate/plate cell. From these observations we construct an « orientation diagram » in the shear rate-concentration plane. It defines three distinct regions where different qualitative and quantitative behaviours are seen (see Fig. 4). At very low shear (region I $\dot{\gamma}<\dot{\gamma}_{1}$ corresponding to line $\mathrm{S}_{1}$ ), the texture looks very inhomogeneous on macroscopic scales $(>10 \mu \mathrm{m})$. A microscopic observation shows that the system is full of defects (which have not been well-characterized) that are moving with the flow (see Fig. 6a).

Above a well-defined shear rate that is a function of the concentration (region II - above line $S_{1}$ - see Fig. 4), the texture observed seems homogeneous. However, the existence of an isotropic modulation of the index of refraction at micrometer scales, is revealed through the clear ring of scattering observed when a laser beam crosses the sample (see Fig. 5). The ring size is an increasing function of the shear rate (Figs. 5a, b and c) and may be associated to a characteristic domain size that decreases continuously from about $10 \mu \mathrm{m}$ to less than $1 \mu \mathrm{m}$ upon increasing shear. With the microscope one effectively sees a well-defined length in the 

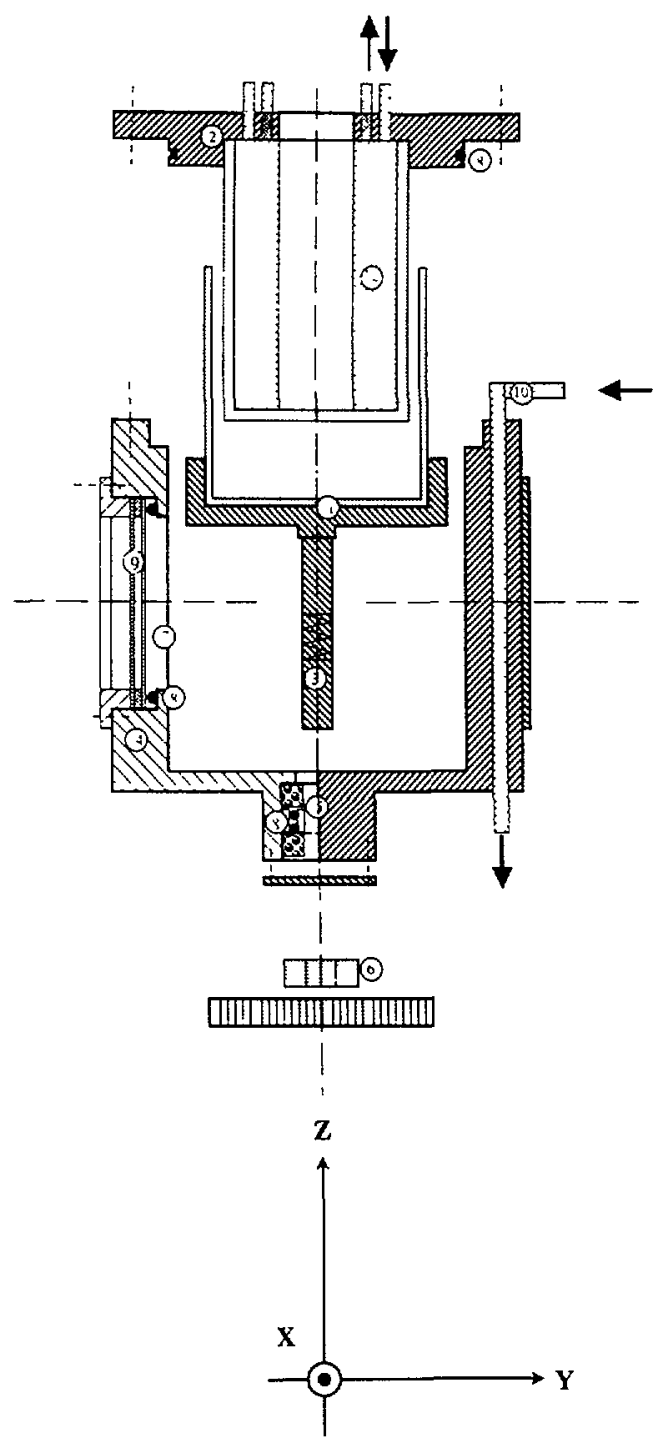

Fig. 2. - Plan of the Couette cell ; 1 corresponds to the rotor, 2 to the stator, 3 is the rotating axis, 4 is the sealed thermostated oven, 5 a sealed rotating ball bearings, 6 a crown wheel to transmit the rotation, 8 seals, 9 Quartz or Glass windows and 10 water circulation coming from a thermostated bath. The Zaxis is the symmetry axis; the $\mathrm{X}$-axis is along the tangential (velocity) direction; the $\mathrm{Y}$-axis is along the radial (velocity gradient) direction.

modulation of the birefringence (Fig. $6 \mathrm{~b}$ and c) that does not exist at lower shear rate (region I, see Fig. 6a). When the shear is suddenly stopped, the ring persists and so does the modulation of the refractive index.

At higher shear rates, the ring finally disappears when the line $S_{2}$ (which defines shear rate $\dot{\gamma}_{2}$ ) is reached (see Fig. 4). Above $\dot{\gamma}_{2}$ (and below $\dot{\gamma}_{3}$, corresponding to line $\mathrm{S}_{3}$ in Fig. 4) a welldefined texture is again visually noticeable, using crossed polarizers or even without polarizers in certain cases. In the Couette cell, the texture is made with alternating dark and bright (using 


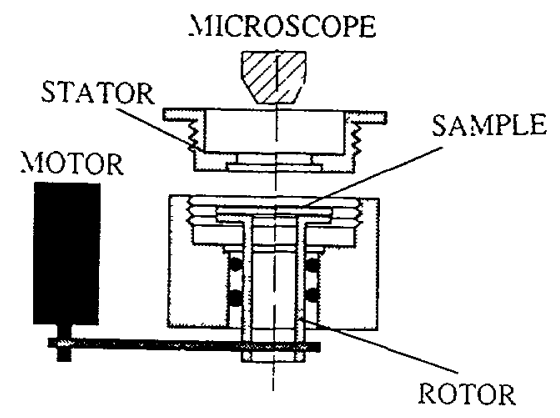

Fig. 3. - Sketchy plan of the plate/plate cell we have used for optical observations (microscopy).
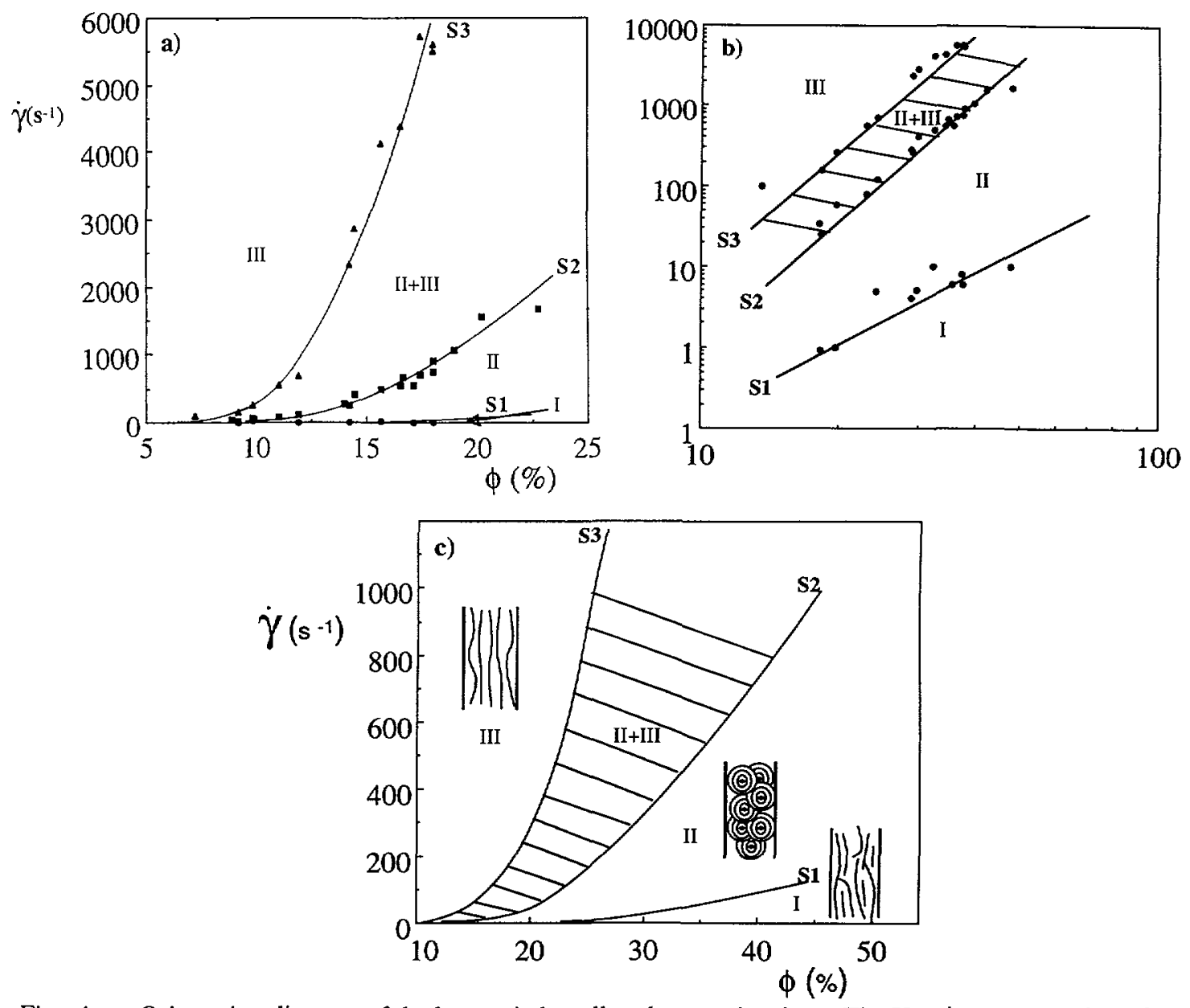

Fig. 4. - Orientation diagram of the lyotropic lamellar phase under shear. The $\mathrm{X}$-axis corresponds to the membrane (water + surfactant) weight fraction. The $\mathrm{Y}$-axis is the shear rate applied in the Couette cell. Regions I, II and III correspond to steady «pure orientations » as described in the text. The region labelled II + III corresponds to a mixed state where states II and III coexist in the cell. Notice that the experiments are done fixing the shear rate : a) is a plot in linear scales; b) is a plot using a double logarithmic scale. The straight lines correspond to slopes respectively equal to 3 (from I to II) and around 5 (from II to III) ; c) schematic representation with a drawing of the orientation states. 


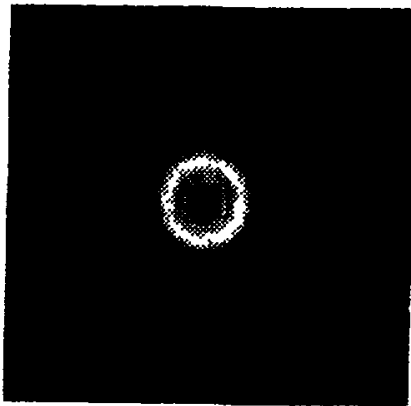

a)

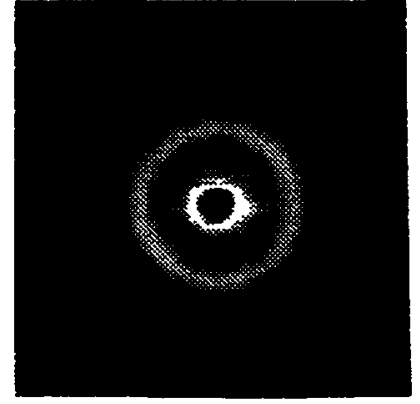

b)

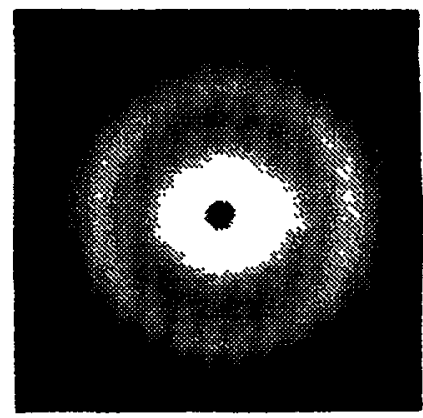

c)

Fig. 5. - Small angle light scattering patterns, observed on a screen. The ring of scattering is observed in region II only. The shear increases from a) to c).

polarizers) band-shaped domains, stacked roughly periodically in equatorial planes : there is an axially-symmetric modulation on macroscopic scales of the refractive index along the Zdirection. The period of the stacking varies with shear rate non monotonically. It first increases with $\dot{\gamma}$ (in the range $0.1-2 \mathrm{~mm}$ ) and then decreases. The modulation disappears when $\mathrm{S}_{3}$ is crossed, i.e. upon entering region III. The microscopic observation has allowed us to conclude that the state between the $S_{2}$ and $S_{3}$ lines is a mixed one that corresponds to the coexistence in alternating stripes of the region II and region III states.

In region III $\left(\dot{\gamma}>\dot{\gamma}_{3}\right)$, the orientation state of the sample looks homogeneous : neither scattering or any microscopic texture is observed. There are no further transitions until the maximum shear rate $\left(\dot{\gamma} \approx 5000 \mathrm{~s}^{-1}\right)$ is reached.

The « orientation diagram », figure 4 , gives the location of the boundaries between regions I, II and III at a given gap. It is clear that the extension of regions I and II decreases when the dilution (or $d$-spacing) increases. Region III is then quickly reached. For instance, a shear rate as low as a few $s^{-1}$ is enough to enter region III for a $d$ around $300 \AA$.

Figure 7 displays the gap size effect on the location of the region boundaries, for gaps 2,1 and $0.5 \mathrm{~mm}$ (resp. Figs. $7 \mathrm{a}, \mathrm{b}$ and $\mathrm{c}$ ). The boundary lines $\mathrm{S}_{1}$ and $\mathrm{S}_{2}$ are basically unaffected however, the location of line $S_{3}$ strongly depends on the gap size (i.e. the smaller the gap size the higher the shear rate $\dot{\gamma}_{3}$ ).

The regions and region boundaries are reproducible and do not depend on the initial state of the system (random or partial orientation; fresh or already used sample). Only the time required to reach a steady state is a function of the history of the sample. In general, the higher the shear rate the faster a steady state is reached. Typical transient times are of the order of 10 to $60 \mathrm{~min}$. When the shear is stopped, the texture remains at first intact. It changes with time depending on the location of the sample respecting regions I, II or III. Indeed, when starting anywhere in region II, there is no noticeable evolution even after days and the ring of scattering can be observed with no changes neither in position nor in intensity (if not sheared again). Moreover, the sample can be removed from the shear cell with care and placed in a flat vessel keeping the same texture (the ring is still visible). The behaviour is different in regions I and III, where states with macroscopic textures differing from the initial ones may be reached in a few hours or less.

\section{Region II : the spherulite state.}

As mentioned above, the state prepared in region II of the orientation diagram is long-lived and remains essentially unaltered after gentle manipulations of the sample. We thus removed part 


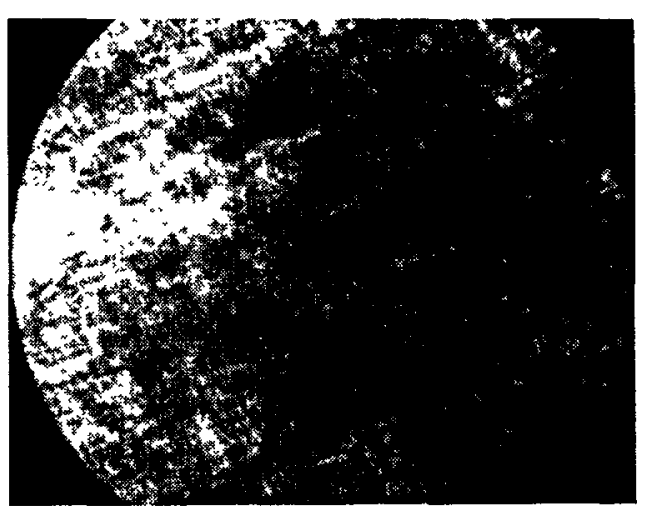

a)

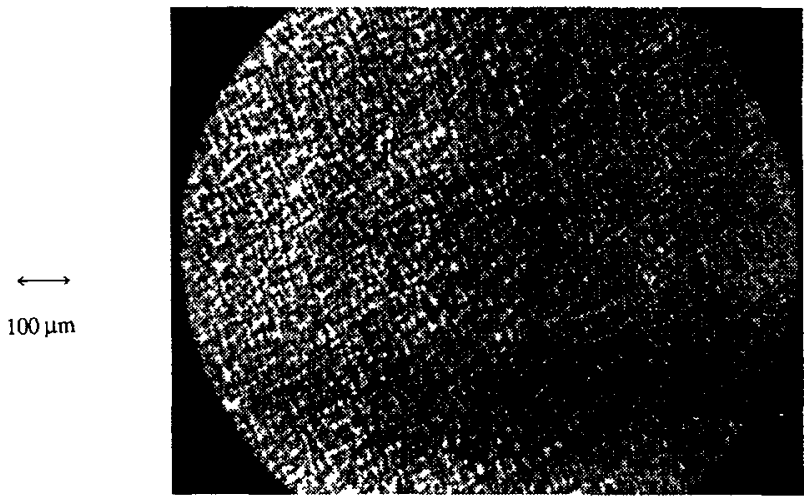

b)

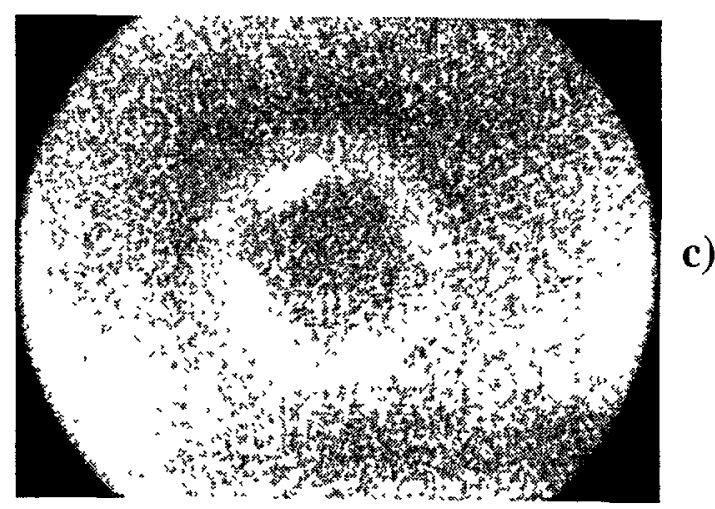

Fig. 6. - Direct space observations under a polarizing microscope. a) Corresponds to a shear rate $<1 \mathrm{~s}^{-1}$ (region I) when $\mathrm{b}$ ) and c) correspond to shear rates in region II (shear higher in c) than in b)).

of the sample from the Couette cell and observed it with a polarizing microscope. A series of observations were made on a given sample prepared in region II, varying the shear rate. A thin isotropic texture with a characteristic length is observed similar to the one observed directly in the plane/plane cell under microscope (Figs. $6 \mathrm{~b}$ and c). The order of magnitude of this length is compatible with the size of the scattering ring. As the shear rate used to prepare the sample is increased, the observed texture becomes thinner and thinner, as expected from the light scattering experiment. The texture does not evolve significantly with time and can be kept for several days in a sealed (to prevent evaporation) cell. We should stress that the texture of a 

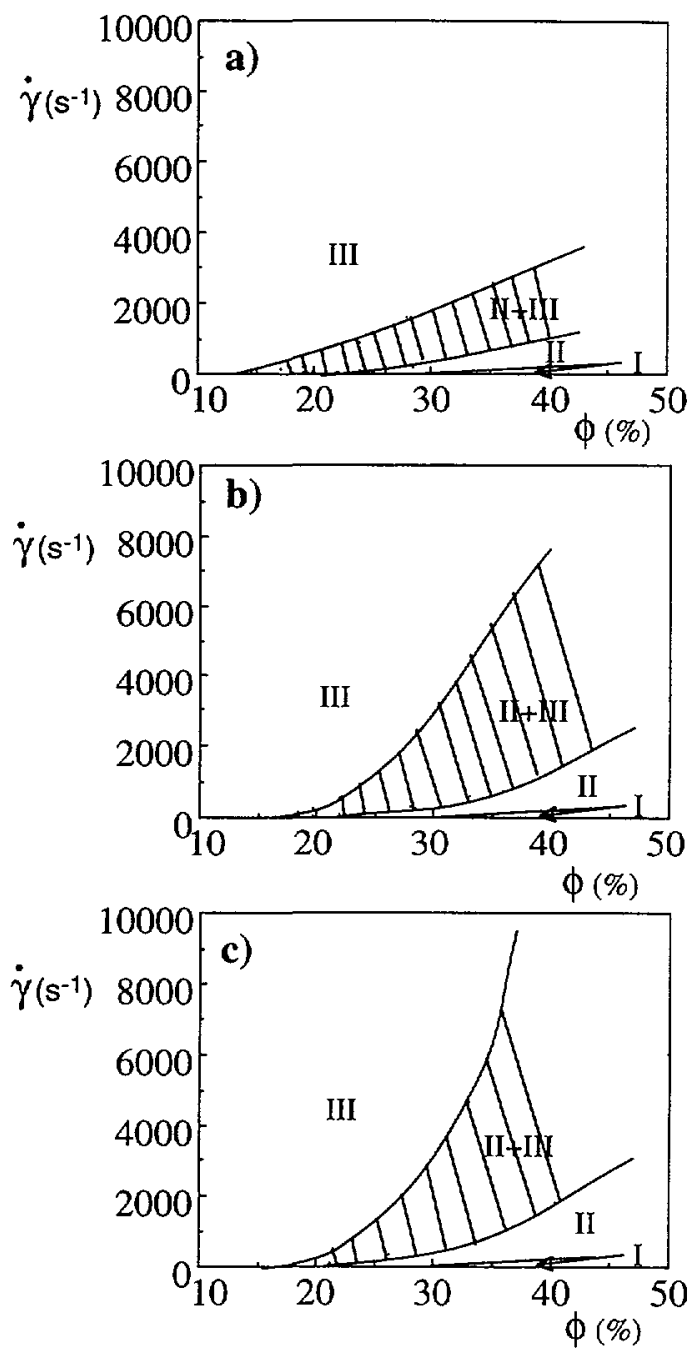

Fig. 7. - Effect of the gap size on the orientation diagram. Gap sizes are respectively equal to of $2 \mathrm{~mm}$ (a), $1 \mathrm{~mm}$ (b) and $0.5 \mathrm{~mm}$ (c). Limits I $\rightarrow$ II and II $\rightarrow$ II + III are more or less insensible to the gap size when the limit from II + III to III varies noticeably.

fresh sample (which has not been previously sheared in a controlled way and consequently with no characteristic length in its texture) placed in the same cell evolves very slowly (within days) to regions of homeotropic orientations separated with focal conics (oily streaks).

In order to further analyse the region II texture, we have done the following experiment. We placed between two microscope slides a small amount of the sheared sample to which we slowly added to one side of the sample a small amount of swelling solvent (9\% pentanol ; $91 \%$ dodecane), in order to get a contact line between the sheared lamellar phase and the pure solvent (see Fig. 8). At the interface, droplets of lamellar phase (spherulites) are observed. Their diameter is equal to the characteristic size of the texture separating from the bulk (see Fig. 9). In fact, it is clearly observed that the bulk sample consists of closely packed spherulites that we are able to separate (swell) using this technique. This observation is central to the new 


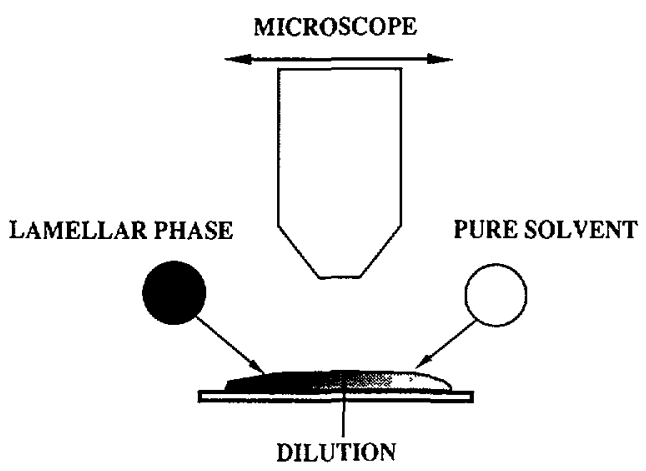

Fig. 8. - Schematic drawing of the contact experiment described in the text. The lamellar phase has been previously sheared in region II and the solvent is the mixture of dodecane (91\%) and pentanol (9\%) that yields the dilution line followed.

a)
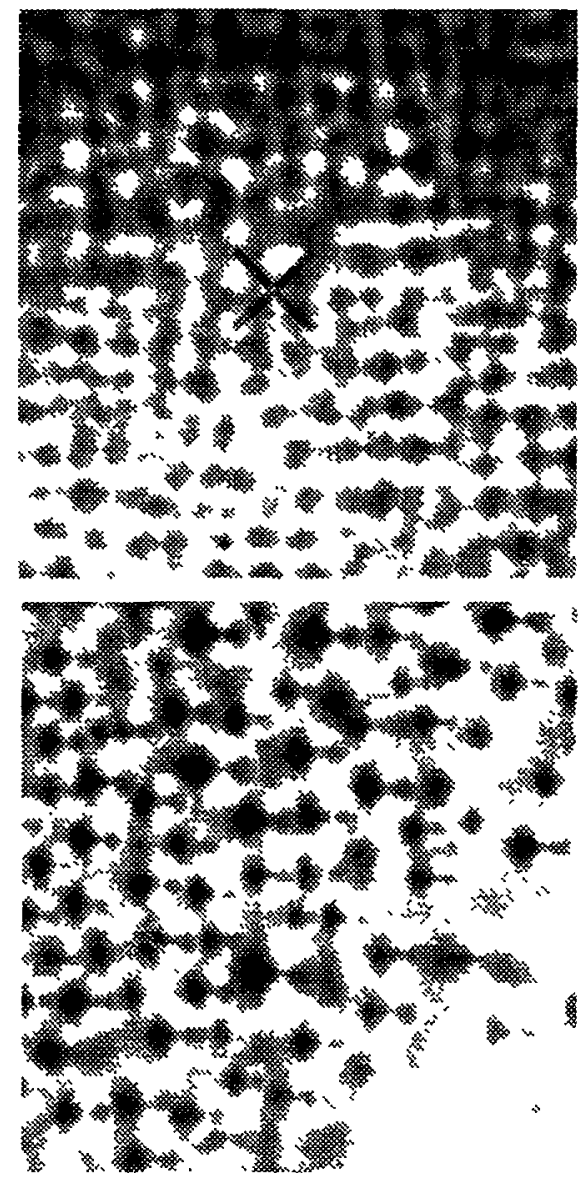

c) b)
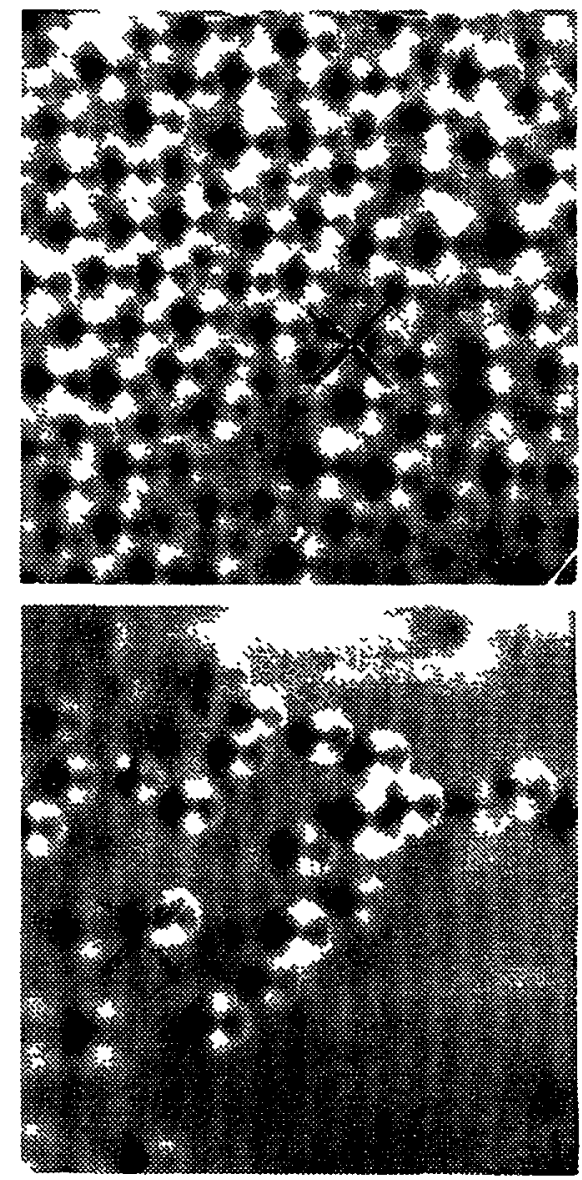

d)

Fig. 9. - Microscopic patterns observed using the contact line method described in figure 8 : a) corresponds to sample situated on the left of the contact line (in the bulk of the lamellar phase); patterns b) to d) correspond to samples situated more and more toward the pure solvent side (on the right). 
way of preparing monodisperse microcapsules by shearing various lamellar phases, described elsewhere $[14,15]$. In the particular case described here (system with SDS, pentanol, dodecane and water) the spherulites dissolve in the solvent after some 20 to $60 \mathrm{~min}$. However in other cases $[14,15]$ depending on the surfactant and solvent chosen, they do not dissolve and remain in suspension (their Brownian motion can be observed when their size is below $5 \mu \mathrm{m}$ ) for several months.

Since a well-defined characteristic size (the spherulite diameter) can be defined by light scattering, we have studied quantitatively its variation as a function of the shear rate at various dilutions of the lamellar phase. A typical curve is shown in figure 10 whereby the size decreases with the shear rate. Anticipating a theoretical interpretation, we plot in figure 11 the spherulite diameter as a function of $\dot{\gamma}^{-1 / 2}$ for samples at different dilutions [14]. A linear behaviour is observed with a dilution-dependent slope (more dilute samples have a lesser slope). We also checked for a possible gap size effect and at high shear rates no such effect was observed. Nevertheless, for small shear rates the spherulite diameter departs from the linear behaviour more strongly for smaller gaps, with sizes larger than predicted (see Fig. 12).

More details may be extracted from light scattering. Indeed, when the sample is sheared the diffraction ring is not perfectly circular but has a slightly elliptic shape. However, when no shear is applied the ring recovers quickly a circular shape. Figure 13 presents the evolution of the ring excentricity (difference between vertical and horizontal radii divided by the mean diameter) as a function of the shear rate. At low shear rate the ring is very nearly circular; at higher shear rate a ten percent excentricity, roughly constant, is observed.

Small-angle neutron scattering experiments have been performed, with the scattering geometry displayed in figure $14:$ the beam is incident radially (along $\mathrm{Y}$-axis), with scattering wave vectors therefore confined to the $X-Z$ plane. It was not possible to get reliable results with a tangential beam (along $X$-axis), mainly because of the strong absorption resulting from the travel through about $10 \mathrm{~mm}$ of sample. Our access to the reciprocal space is thus restricted to the neutral/velocity directions. This restriction is removed in the X-ray scattering experiments made by Safinya et al. [16] on lamellar samples sheared in region III.

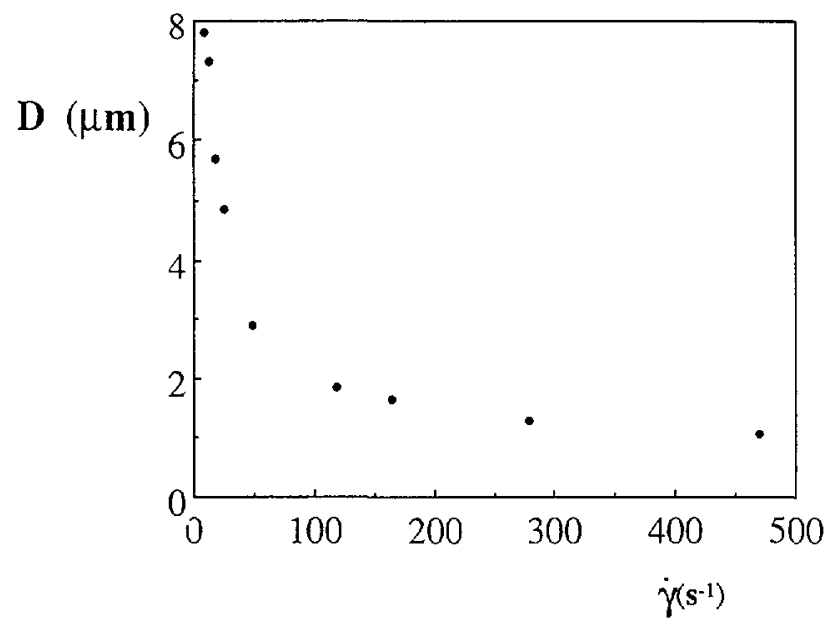

Fig. 10. - Variation of the characteristic size of the refractive index modulation for a lamellar phase sheared in region II. The size is measured using the position of the peak obtained in small angle light scattering (see Fig. 5). It corresponds to the onion size that can be characterized independently by the experiments described in figures 8 and 9. 


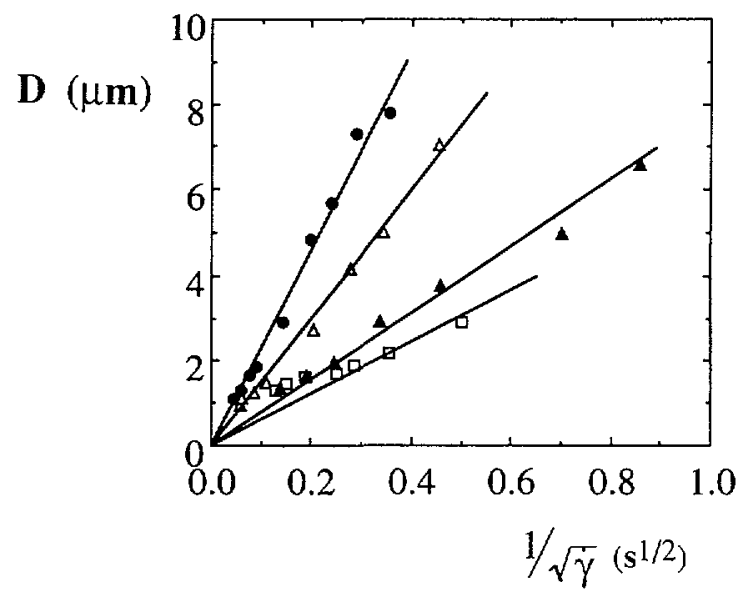

Fig. 11. - Plot of the characteristic onion size (in a Couette cell with a $1 \mathrm{~mm}$ gap) as a function of the inverse of the square root of the shear rate. Different samples have been regrouped in this figure, corresponding to different dilutions. The membrane weight fraction is respectively : $47.6 \%$ (full circles), $42 \%$ (open triangles), $27 \%$ (full triangles), $20 \%$ (open squares). A linear behaviour is observed in each case.

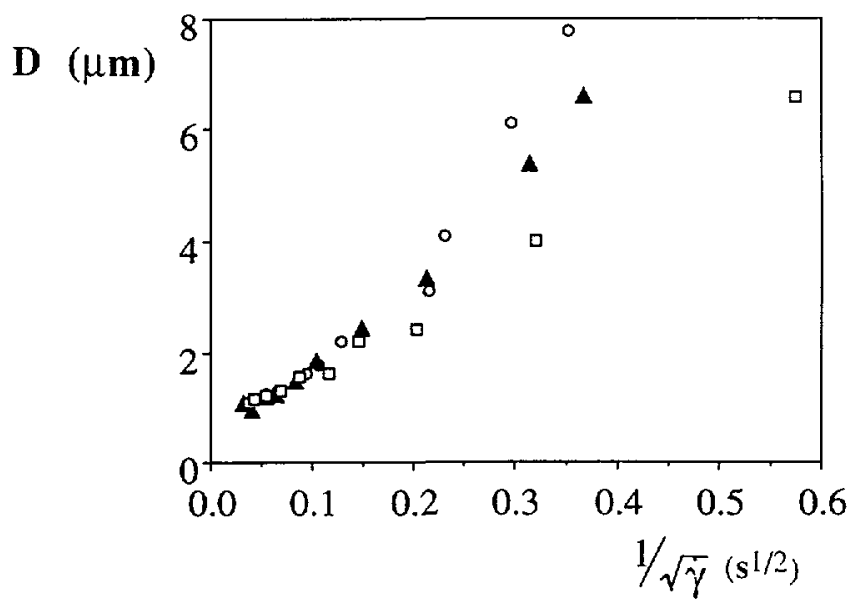

Fig. 12. - Evolution of the characteristic onion size as a function of the inverse square root of the shear rate for a given sample (membrane weight fraction $42 \%$ ), using different gap sizes: open circles correspond to a gap size of $0.5 \mathrm{~mm}$, full triangles to $1 \mathrm{~mm}$ and open squares to $2 \mathrm{~mm}$. At high shear rates sizes are gap-independent but at lower shear rates a systematic deviation from the linear behaviour is observed, more pronounced for the smaller gaps.

Representative results (sample with membrane volume fraction $37 \%$ ) are displayed in figure 15 . The scattered intensity is represented by level lines in the $Q_{x}-Q_{z}$ plane. In region $I$, the scattering is clearly anisotropic (Fig. 15a) and becomes isotropic when region II is reached (Fig. 15b). When the shear rate is further increased (still in region II), there is some weak anisotropy (Fig. 15c). A strong anisotropy, figure 15d, characterizes region III.

The information that we gain from the spectra, mainly through the orientation and intensity of the Bragg diffraction, corresponds to the membrane orientation distribution. In region II, as 


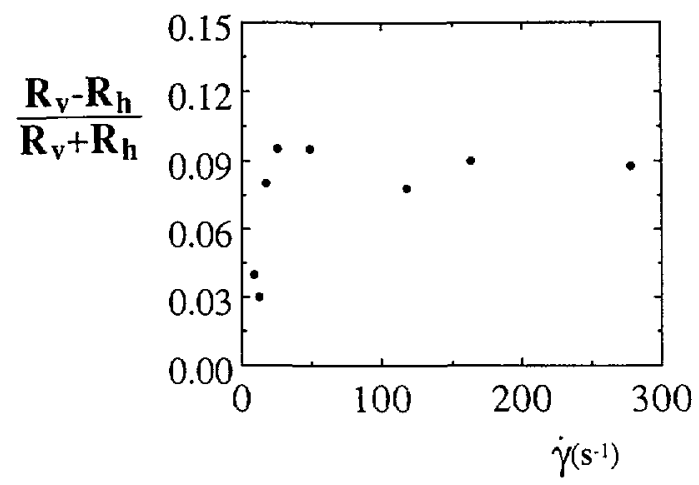

Fig. 13. - Evolution of the ellipticity of the ring observed in the light scattering experiments (see Fig. 5) as a function of the shear rate in the region II. The ellipticity is quantified by the ratio between the difference between the two diameters of the ring (vertical-horizontal) divided by their sum. The ellipticity is first very small (at low shear rates) but increases to saturate to a value typically equal to ten percent.

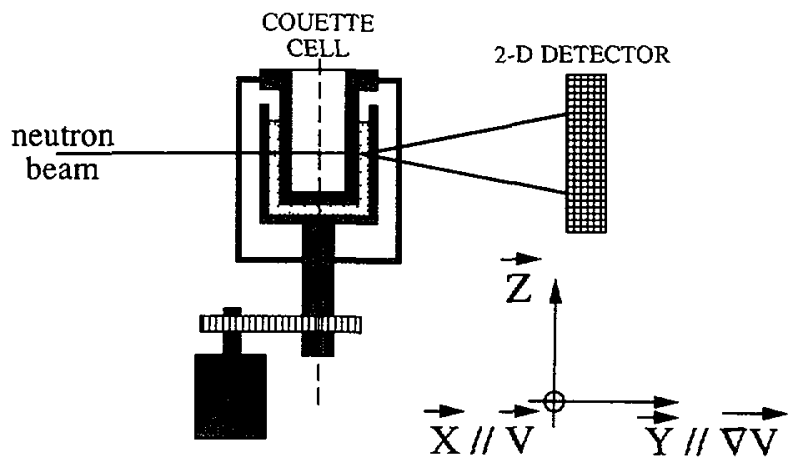

Fig. 14. - Geometry used in the neutron scattering experiments (PAXY beam line at laboratoire LéonBrillouin, Saclay).

detailed in figure 16, we have first at very small shear rates an isotropic (in the $\mathrm{Q}_{\mathrm{X}}-\mathrm{Q}_{\mathrm{z}}$ plane) Bragg diffraction (Fig. 16a). This is compatible with the onion model of the spherulites, where membranes are wrapped in successive, equidistant layers on a spherical core. As the shear rate increases some anisotropy builds up in the Bragg diffraction (Figs. 16b, $\mathrm{c}$ and $\mathrm{d}$ ). This corresponds to the appearance of an elliptic light scattering ring. We propose that the spherulites (spherical on average at low shear) become elongated in a way shown schematically in figure 17. This elongation is of the order of $10 \%$ of the total size.

\section{Region III : the oriented state.}

The orientation state of lamellar samples sheared in region III has been determined with both neutron scattering and birefringence measurements (conoscopy). These techniques provide access to the membrane orientation about the smectic stacking and macroscopic scales.

Only rather dilute samples (oil mass fraction ranging from $55 \%$ to $78 \%$ ) may be prepared in region III, because more concentrated ones require too high a shear rate to enter this region. A typical neutron scattering profile (oil content $60 \%$ ) is given in figure $15 \mathrm{~d}$. This profile 
a)

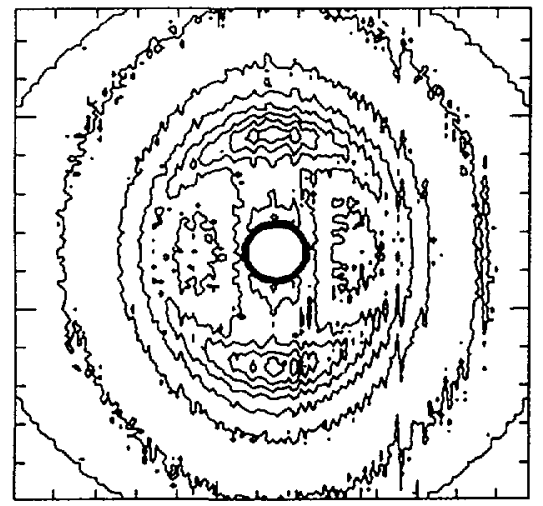

c)

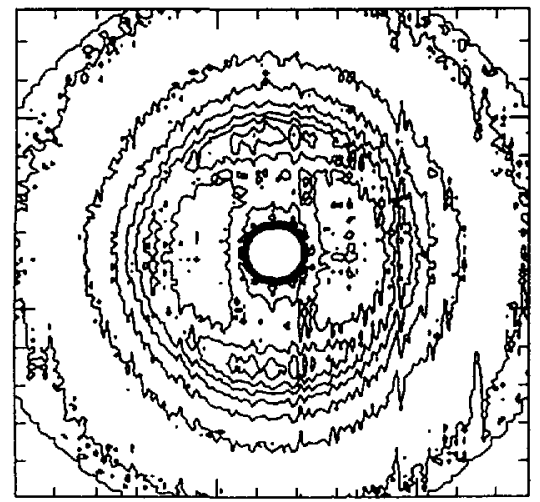

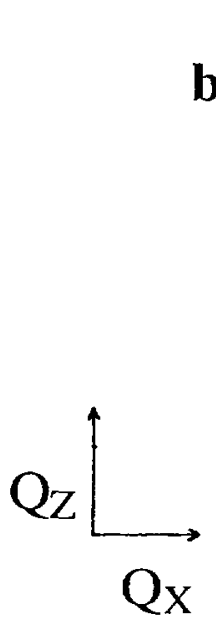

d)

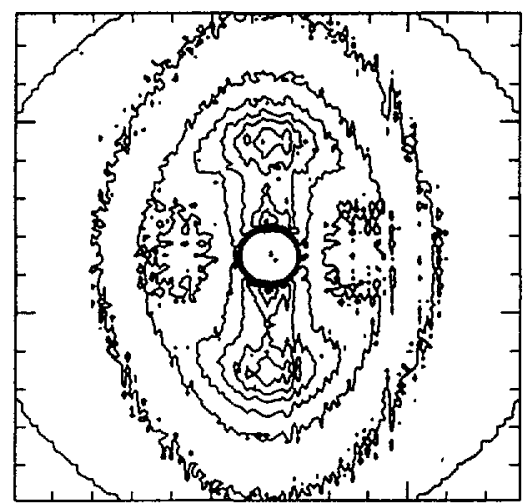

Fig. 15. - Typical two dimensional spectra obtained using neutron scattering : a) corresponds to region I $\left(\dot{\gamma}=1 \mathrm{~s}^{-1}\right) ; \mathrm{b}$ ) and c) to region II (respectively $\dot{\gamma}=24 \mathrm{~s}^{-1}$ and $\dot{\gamma}=127 \mathrm{~s}^{-1}$ ); d) corresponds to region III $\left(\dot{\gamma}=3500 \mathrm{~s}^{-1}\right)$.

$I(q)$ is characteristic of at least partially oriented dilute lamellar phases exhibiting both small angle scattering and a quasi-Bragg peak [8]. The scattering along $\mathrm{Q}_{\mathrm{X}}$, the (tangential) flow direction, is negligible indicating that there are no membranes perpendicular to the flow. The scattering along $\mathrm{Q}_{Z}$ (neutral direction) originates in the membranes that are perpendicular to this direction. However, since the intensity along $Q_{Z}$ remains constant at the level measured in region II where the scattering from a perfect powder is expected, most of the membranes are probably oriented with their normal along the (radial) $Q_{Y}$ direction. This hypothesis is well supported by the conoscopy experiments described below. Finally, we note that for the most dilute samples (highest shear rates), the intensity of the Bragg peak along $Q_{Z}$ may decrease slightly.

BIREFRINGENCE MEASUREMENTS. - The smectic A phase is a uniaxial medium and therefore birefringent in general. The polarization state of the light transmitted through an oriented sample depends on the birefringence of the system $(\Delta n)$ and on the orientation of the incident wave vector with respect to the optical axis. Consequently, the analysis of the intensity of the light transmitted between polarizers gives information on the orientation of the system.

Let $\beta$ be the angle between the incident light wave vector $\mathbf{k}$ and the optical axis $\mathbf{n}$ (which is also the membrane normal), with $\theta$ the angle between the incident polarization direction and a 
a)
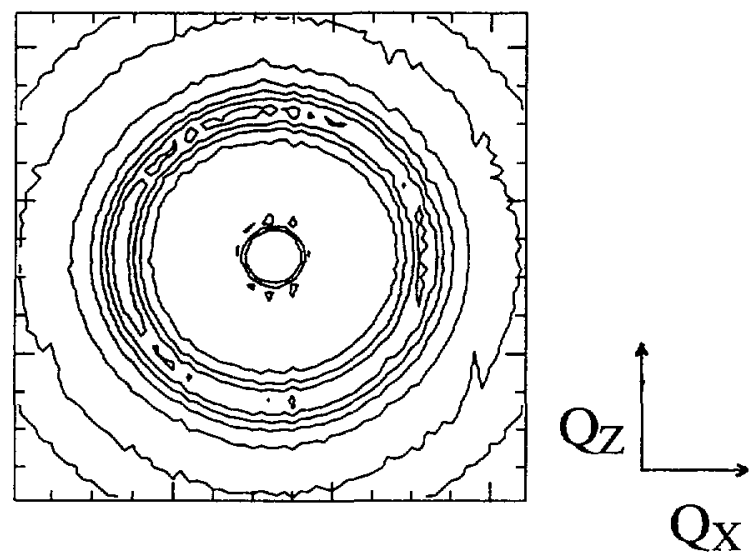

b)
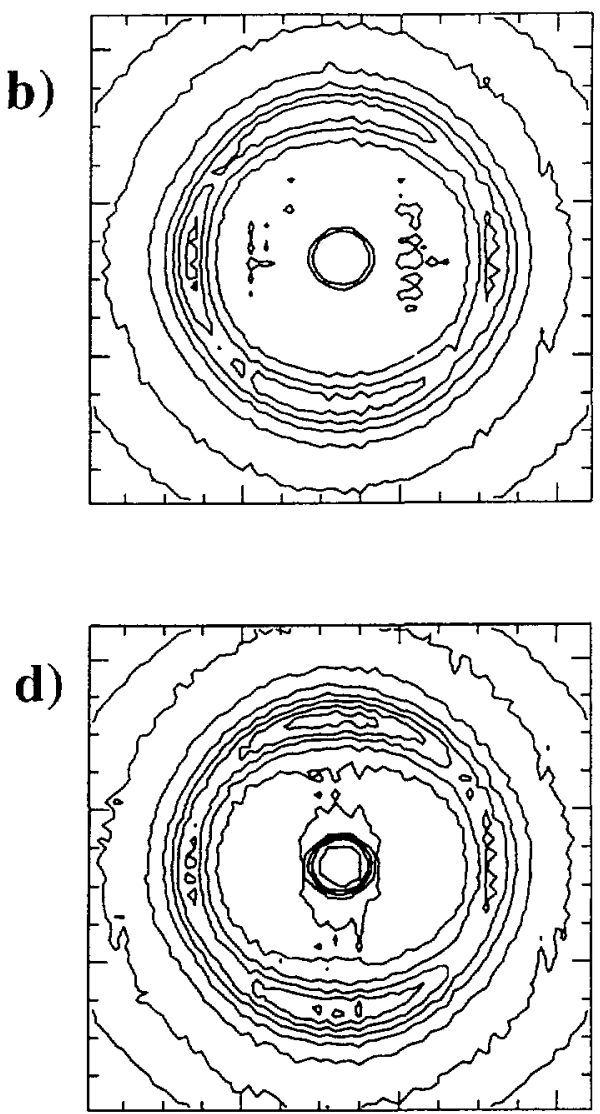

c)

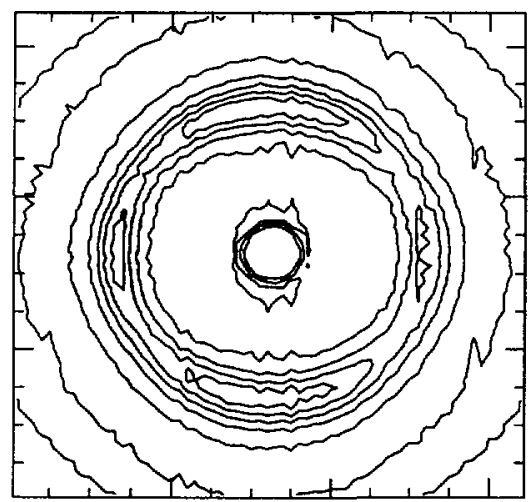

Fig. 16. - Neutron scattering patterns observed in region II, for a membrane weight fraction of $48 \%$. The respective shear rates are $\dot{\gamma}=8 \mathrm{~s}^{-1}$ (a), $\dot{\gamma}=255 \mathrm{~s}^{-1}$ (b), $\dot{\gamma}=490 \mathrm{~s}^{-1}$ (c) and $\dot{\gamma}=1200 \mathrm{~s}^{-1}$ (d).

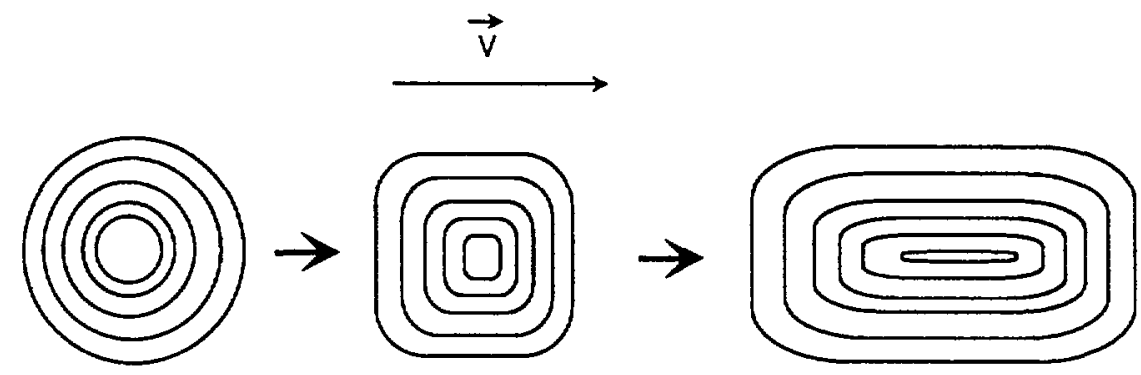

Fig. 17. - Schematic interpretation of the anisotropy observed in both light and neutron scattering (Figs. 13 and 16). The onions are slightly deformed by the shear however, this elongation remains weak (of the order of $10 \%$ ). 
reference direction perpendicular to both $\mathbf{n}$ and $\mathbf{k}$ (see Fig. 18). The relative intensity measured (after an analyzer making an angle $\phi$ with respect to the polarizer) for a light beam travelling a thickness $e$ through the sample is given by the standard formula:

$$
\frac{I}{I_{0}}=\cos ^{2} \phi-\sin 2 \theta \sin [2(\theta+\phi)] \sin ^{2}\left[\frac{\pi e}{\lambda} \Delta n \sin ^{2} \beta\right]
$$

if one may assume that the birefringence $\Delta n$ is small compared to both ordinary or extraordinary indices.
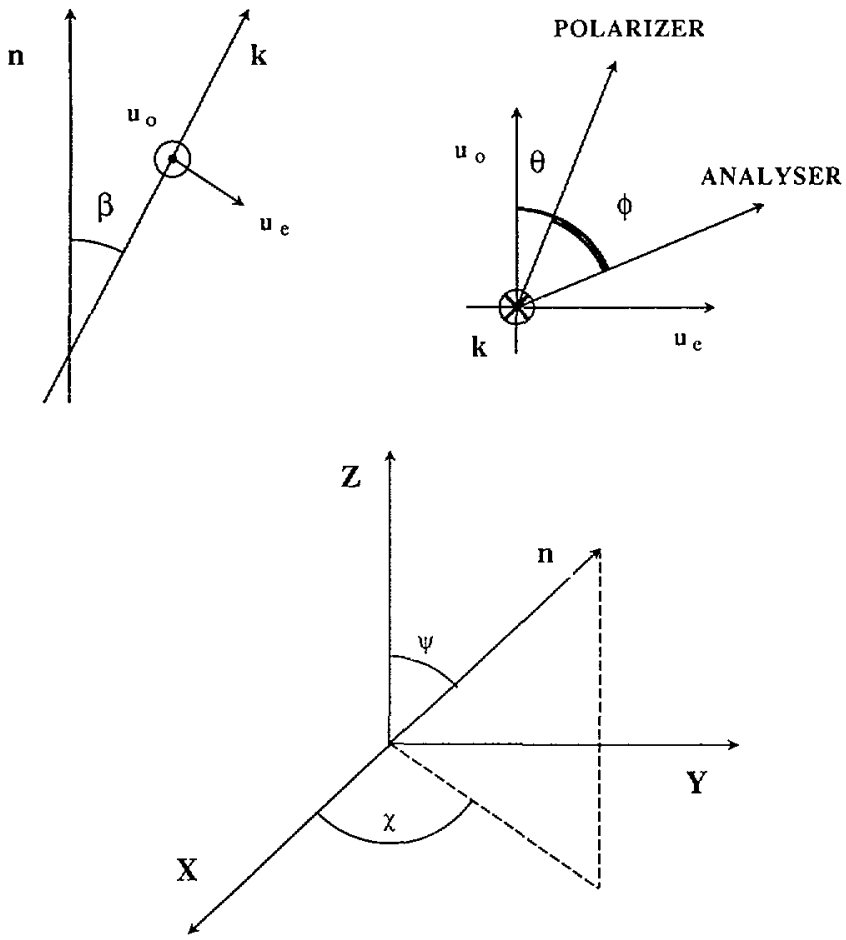

Fig. 18. - Definitions of the angles used in the conoscopy experiment. The angle between the membrane normal $\mathbf{n}$ and the wave vector of the light $\mathbf{k}$ is $\beta$; the two angles $\theta$ and $\phi$ refer to the polarizer and analyser orientations, respectively; the two angles $\psi$ and $\chi$ define the orientation of the membrane normal $\mathbf{n}$ in the Couette system of axes.

This equation has a simpler form when polarizer and analyzer are at right angles $(\phi=\pi / 2)$ :

$$
\frac{I}{I_{0}}=\sin ^{2} 2 \theta \sin ^{2}\left[\frac{\pi e}{\lambda} \Delta n \sin ^{2} \beta\right]
$$

For simple orientations of the lamellar phase in the Couette cell, with light propagating along the $Y$-axis (see Fig. 19), 

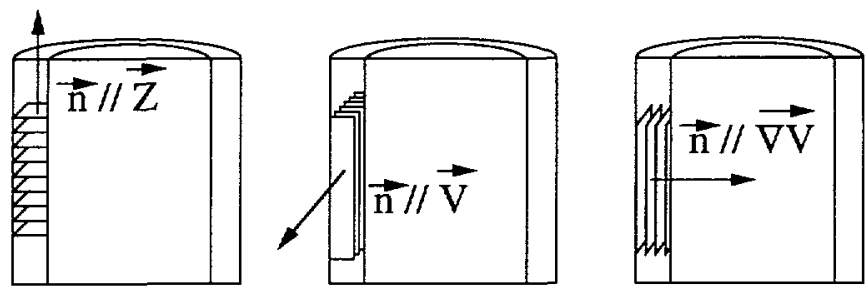

Fig. 19. - Schematic representation of the simple (« pure ») orientations of a smectic layered system in a flow. The different states are respectively labelled as $\mathrm{A}(\mathbf{n} / / Z), \mathrm{B}(\mathbf{n} / / \mathbf{v})$ and $\mathrm{C}(\mathbf{n} / / \nabla \mathbf{v})$.

orientations $\mathrm{A}$ or $\mathrm{B}\left(\beta=\frac{\pi}{2}\right)$ lead to:

$$
\frac{I}{I_{0}}=\sin ^{2}(2 \theta) \sin ^{2}\left(\frac{\pi}{\lambda} \Delta n e\right)
$$

orientation $\mathrm{C}(\beta=0)$ leads to:

$$
\frac{I}{I_{0}}=0
$$

In order to determine the birefringence $\Delta n$, we have measured the intensity transmitted through samples of known orientation. Such samples are prepared in thin rectangular glass capillaries ( $2 \mathrm{~mm}$ wide, $200 \mu \mathrm{m}$ thick), where a good homeotropic orientation is achieved with a thermal treatment. The transmission of a polarized $(\theta=\pi / 4) \mathrm{He}-\mathrm{Ne}$ laser beam ( $\lambda=6328 \AA$ ) is measured as a function of the angle $\alpha$ between the incident light and the normal to the capillary (see Fig. 20). Because of the homeotropic orientation $\beta=\alpha$, the transmitted light with crossed polarizers $(\phi=\pi / 2)$ is given by :

$$
\frac{I}{I_{0}}=\sin ^{2}\left[\frac{\pi}{2} \Delta n\left(\frac{e}{\cos \alpha}\right) \sin ^{2} \alpha\right]
$$

where $e$ is now the thickness of the capillary (optical path $e / \cos \alpha$ ). Fits of the data to equation (4), shown in figure 21 , give the birefringence as a function of the membrane weight fraction $\phi_{\mathrm{m}}$, figure 22 . The birefringence $\Delta n$ varies linearly with $\phi_{\mathrm{m}}$ and we get :

$$
\Delta n=a+b \phi_{\mathrm{m}}
$$

with

$$
a=-1.055 \times 10^{-4} \text { and } b=4.91 \times 10^{-3}
$$

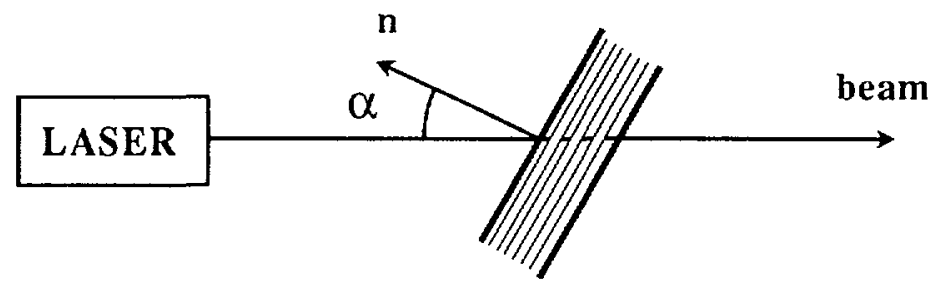

\section{CAPILLARY}

Fig. 20. - Schematic drawing of the set-up used to measure the birefringence of a series of samples perfectly aligned in capillaries. The intensity transmitted between crossed polarizers is recorded as a function of the angle $\alpha$. 

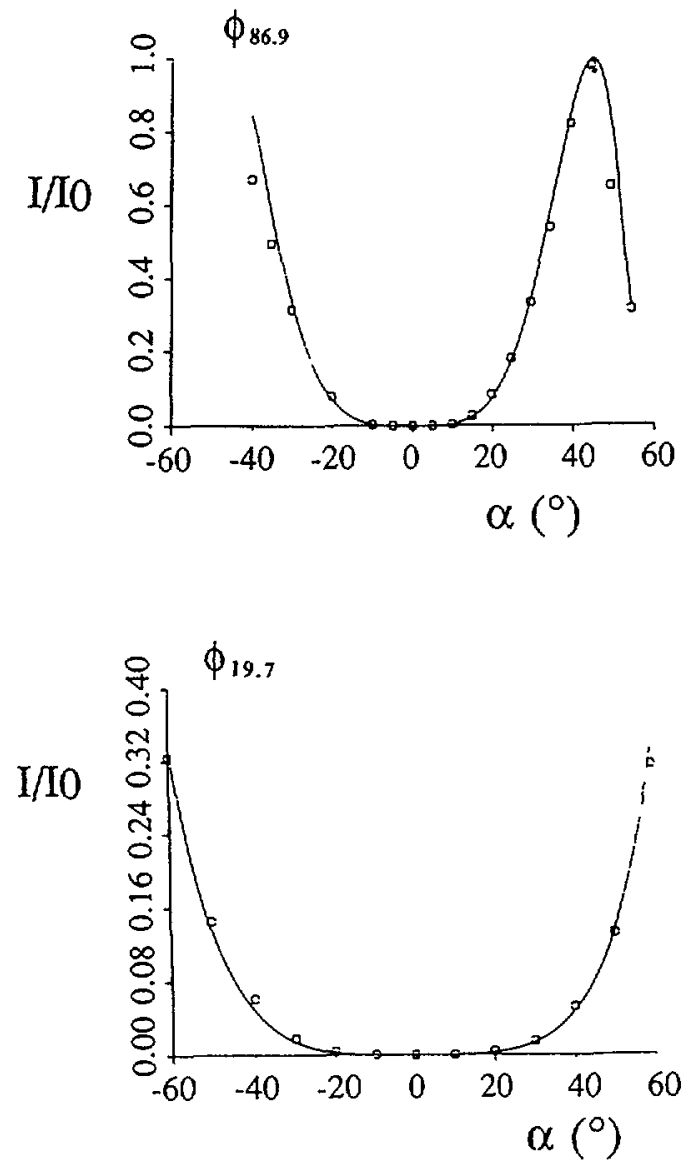

Fig. 21. - Typical transmission curves obtained using the set up represented figure 20. The full lines are obtained by fitting the experimental points to the theoretical expression, equation (4).

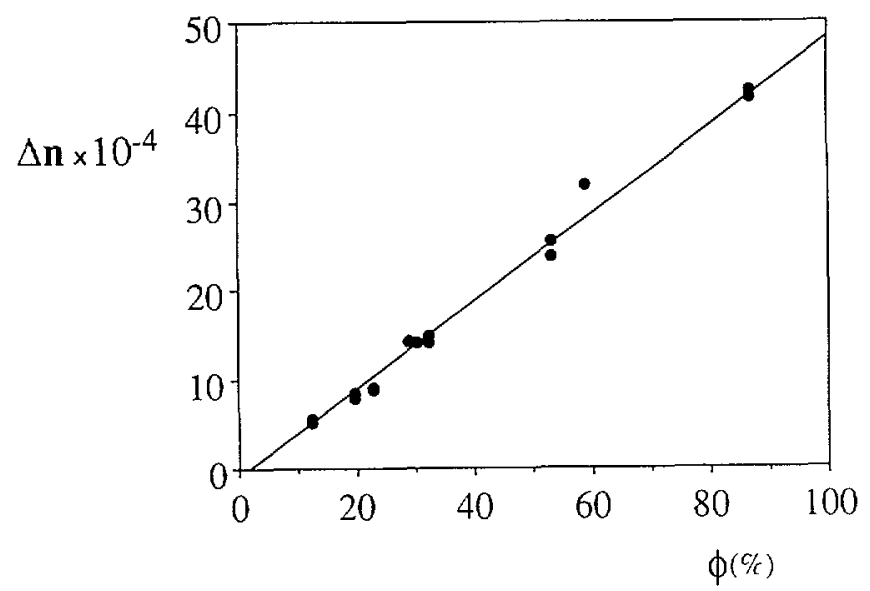

Fig. 22. - Values of birefringence obtained for samples with different membrane weight fractions $\phi$. The behaviour is basically linear and the best fit corresponds to:

$$
\Delta n=a+b \phi \text {, with } a=-1.055 \times 10^{-4} \text { and } b=4.91 \times 10^{-3}
$$


The same technique is now used to determine the orientation (knowing the birefringence) of a lamellar phase under shear. The experimental set-up allows to send the beam through one gap only (see Fig. 23). It also allows the angle $\alpha$ (between the incident beam and the $Y$-axis) to vary. In order to simplify the analysis and to optimize the intensity, the polarization is rotated $\pi / 4$ away from the $Z$-axis/beam plane and the analyser set at right angle $(\phi=\pi / 2)$. We have checked that the maximum intensity is effectively obtained with such a geometry.

Two types of experiments have been performed. On one hand, we have recorded the intensity for $\alpha=0$ as a function of the shear rate. Some typical data are shown in figure 24 . The intensity first increases as a function of the shear rate when the sample goes through regions I and II, then saturates in region III. As for the neutron scattering, in the case of the most dilute sample the intensity slightly decreases at large shear rates. When the birefringence is high enough, the ratio between the measured intensity and incident one may oscillate in

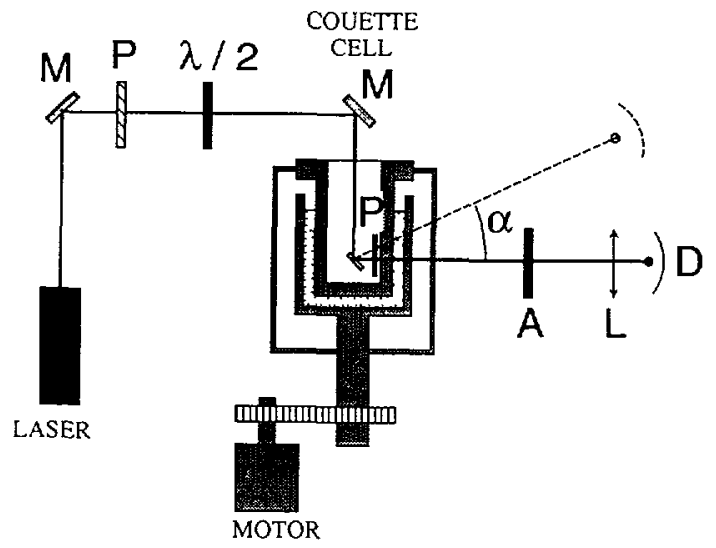

Fig. 23. - Schematic drawing of the set-up used to determine the orientation of a sample of known birefringence but unknown orientation when it is sheared in the Couette cell. The angle $\alpha$ is varied by a small rotating mirror placed inside the stator.

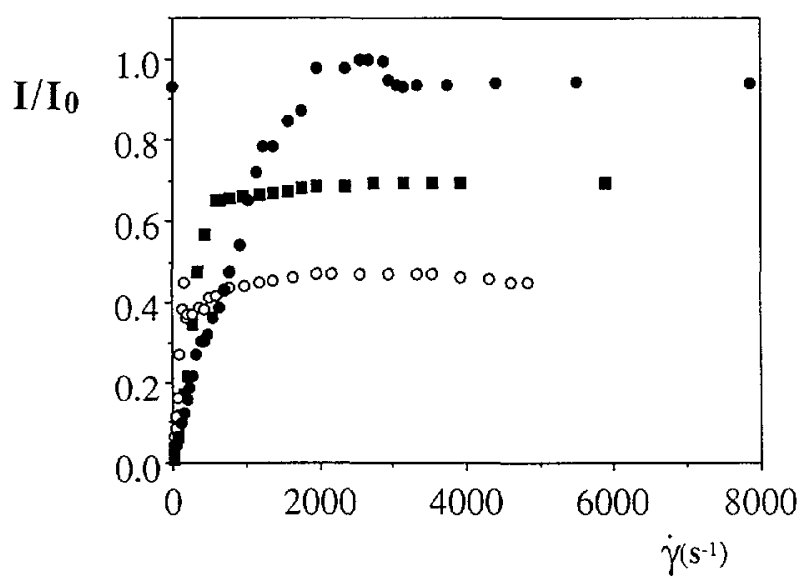

Fig. 24. - Variation of the intensity transmitted between crossed polarizers through different samples, as a function of the shear rate, for normal incidence $(\alpha=0)$. The plateau corresponds to the appearance of region III. 
region II + III before reaching its plateau value in region III. For the most concentrated sample several oscillations can be seen (Fig. 25). This effect is easily understood in terms of an increase of the phase term beyond $\pi / 2$, in equation (2). This first experiment confirms the results obtained with neutron scattering, namely that region III corresponds to a constant orientation (except for the most dilute sample where a slight evolution exists ; we come back on that point later on).

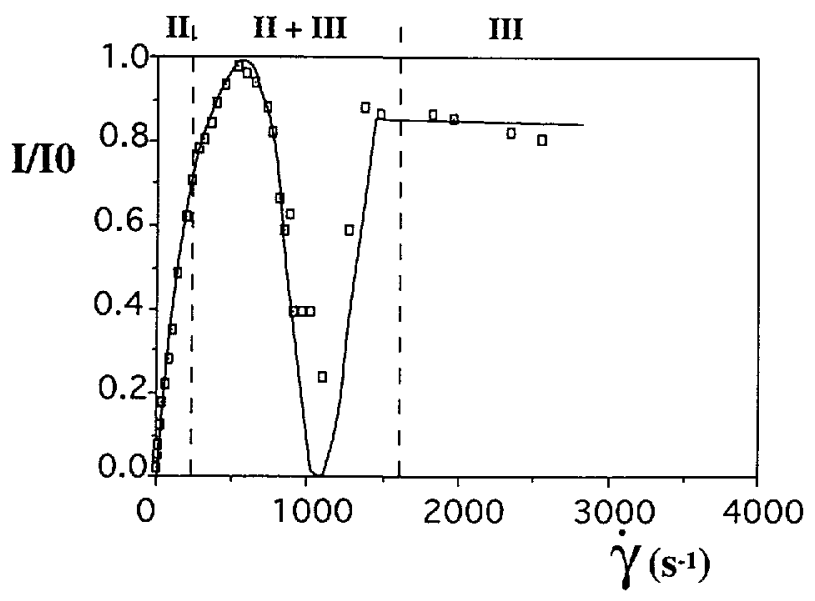

Fig. 25. - Same as previous figure for a given concentrated sample. The oscillations are due to the fact the phase becomes larger than $\pi / 2$.

We then analyze the orientation in region III, in a second experiment where we vary the incident angle (around normal incidence), with a shear rate fixed in the plateau region, for different dilutions. From the neutron scattering experiment we know that there are no domains having the membrane normal along the $\mathrm{X}$-axis, i.e. parallel to the flow. We thus describe the orientation state in terms of domains oriented in the $\mathrm{Y}-\mathrm{Z}$ plane. The model we use assumes a Gaussian orientation distribution with width $\Delta \psi$, centered around the $\mathrm{C}$ orientation (see Fig. 19 ; membrane normal $\mathbf{n}$ parallel to the radial, or velocity gradient axis). This leads (see appendix) to the following transmitted intensity :

$$
\frac{I}{I_{0}}=\sin ^{2}\left[\frac{\pi \Delta n e}{\lambda}\left\{\Delta \psi^{2}+\alpha^{2}\left(1-\frac{3 \Delta \psi^{2}}{2}\right)\right\}\right]
$$

for small values of $\alpha$.

Two quantities, namely the birefringence $\Delta n$ and the mosaic $\Delta \psi$, may be independently extracted in principle from the data. Plots of arcsin $\left[\left(I / I_{0}\right)^{1 / 2}\right]$ as a function of $\alpha^{2}$, with linear fits are presented in figure 26 , with the resulting values for the two parameters given in table I. The birefringence values are consistently very close to the ones determined on oriented samples. The evolution with dilution of the mosaic angle $\Delta \psi$ is given in figure 27 . Its value seems to be constant and of the order of $25^{\circ}$ except for the most dilute samples, which give higher $\Delta \psi$ values at their lowest region III shear rates. However as we have already noticed, for most dilute samples there is an evolution of the Bragg peak intensity corresponding to a change of orientation in region III, as shear is increased. The conoscopy analysis indicates that the mosaic angle, initially high, comes back to about $25^{\circ}$ at higher shear rates (see Fig. 28). 

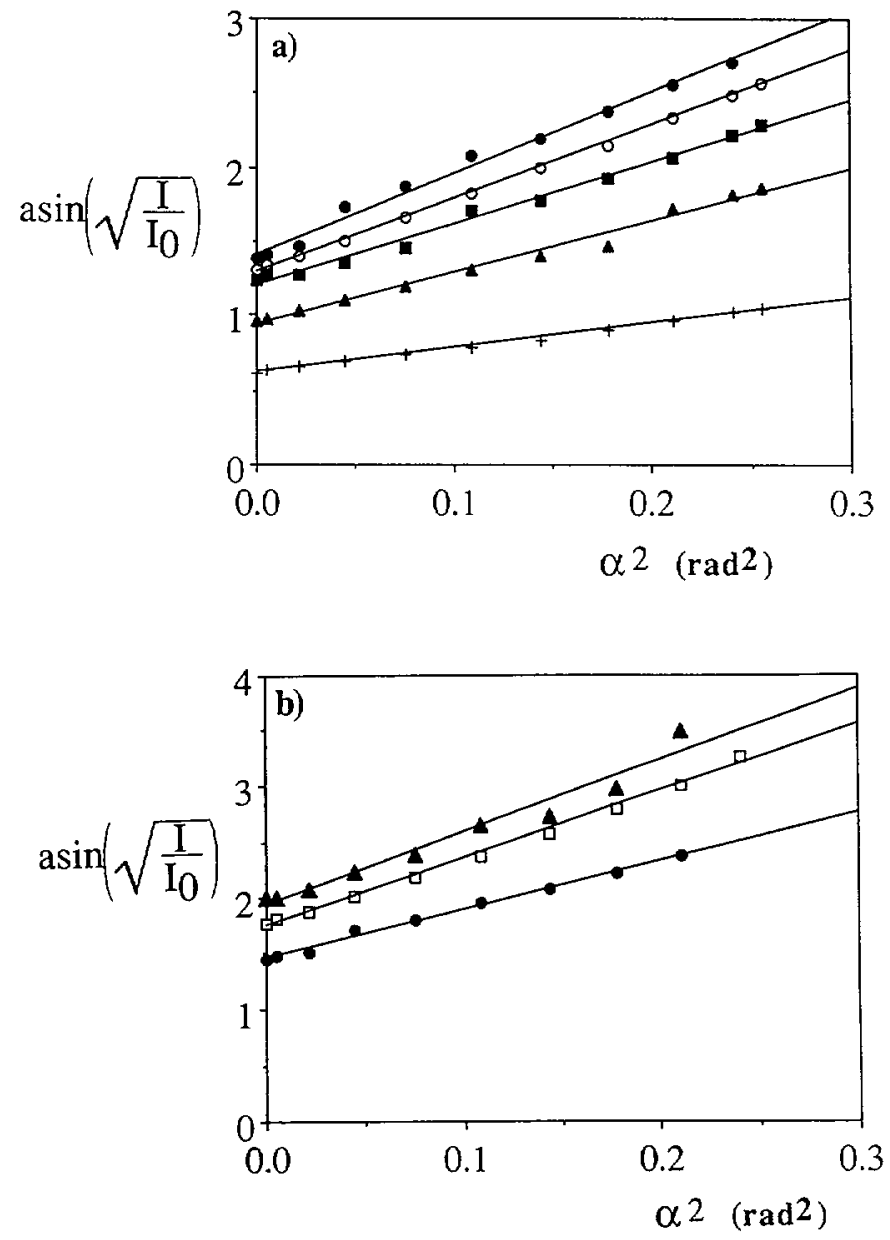

Fig. 26. - Evolution of the normalized intensity as a function of the square of the angle $\alpha$ for small $\alpha$ s. Knowing the birefringence, the slope allows to get the mosaic angle using equation (6).

The same kind of model has been used in analyzing the neutron scattering intensities, comparing the Bragg intensities for a perfect powder sample (obtained in region II) and for a sample in region III, assuming again a Gaussian mosaicity around the direction of the velocity gradient. We found a width in the 30 to $45^{\circ}$ range, in agreement with the conoscopy measurements.

\section{Discussion.}

From the results we obtained, we conclude that as shear increases three types of orientation are seen. At low and high shear rates, the dominant orientation of the smectic domains is the $\mathrm{C}$ orientation and corresponds to the membrane normal pointing along the radial velocity gradient direction. The main difference between the low (region I) and high (region III) shear regions is that their mosaicities are quite different. In region I, there are domains oriented along the flow as well as along the Z-axis, while in region III the phase is better oriented with a negligible mosaic in the direction of the flow and about $25^{\circ}$ disorientation with respect to a pure $\mathrm{C}$ 
Table I. - Values of parameters for the linear fit to the curves shown in figure 26. The values of the birefringence and mosaic angle are calculated using equation (6). The comparison between different gap sizes ( 1 and $2 \mathrm{~mm}$ ) is given.

\begin{tabular}{|l|l|l|l|l|}
\hline$\phi_{\mathrm{X}}(\%)$ & \multicolumn{1}{|c|}{$\Delta \mathrm{n}(1 \mathrm{~mm})$} & $\Delta \psi\left({ }^{\circ}\right)(1 \mathrm{~mm})$ & $\Delta \mathrm{n}(2 \mathrm{~mm})$ & $\Delta \psi\left({ }^{\circ}\right)(2 \mathrm{~mm})$ \\
\hline 39.98 & $19.20 \times 10^{-4}$ & 22.05 & & \\
\hline 35.49 & $17.25 \times 10^{-4}$ & 22.26 & & \\
\hline 29.67 & $16.21 \times 10^{-4}$ & 22.80 & & \\
\hline 28.76 & $14.25 \times 10^{-4}$ & 23.63 & $11.01 \times 10^{-4}$ & 24.13 \\
\hline 24.45 & $12.23 \times 10^{-4}$ & 22.43 & & \\
\hline 22.24 & $8.40 \times 10^{-4}$ & 27.4 & $10.26 \times 10^{-4}$ & 23.75 \\
\hline 19.13 & $12.24 \times 10^{-4}$ & 22.49 & & \\
\hline 16.99 & & & $7.28 \times 10^{-4}$ & 25.74 \\
\hline 13.54 & $5.20 \times 10^{-4}$ & 28.05 & & \\
\hline 9.45 & $3.04 \times 10^{-4}$ & 40.75 & & \\
\hline
\end{tabular}

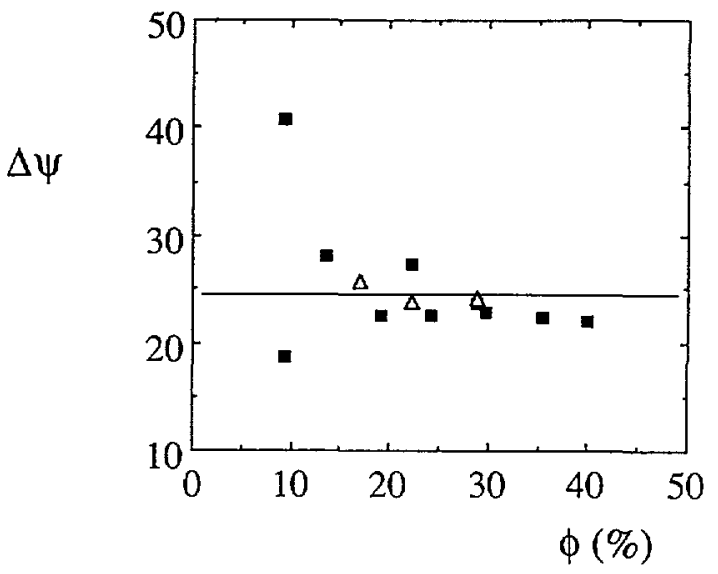

Fig. 27. - Evolution of the mosaic angle obtained using equation (6) as a function of the membrane weight fraction in region III. Full square corresponds to a $1 \mathrm{~mm}$ gap and triangles to $2 \mathrm{~mm}$.

orientation. One of the most striking result is the existence of a completely isotropic orientation in an intermediate regime of shear rates: in region II, the membranes are wrapped around a spherical core forming large objects (the spherulites) that are close-packed and with their size determined by the shear rate. These different homogeneous states of orientation are separated with dynamic (out-of-equilibrium) transitions. The nature of such transitions is under investigation however, it is clear that going from region II to region III involves a first order transition : a coexisting region is clearly seen in between. The complete understanding of this 

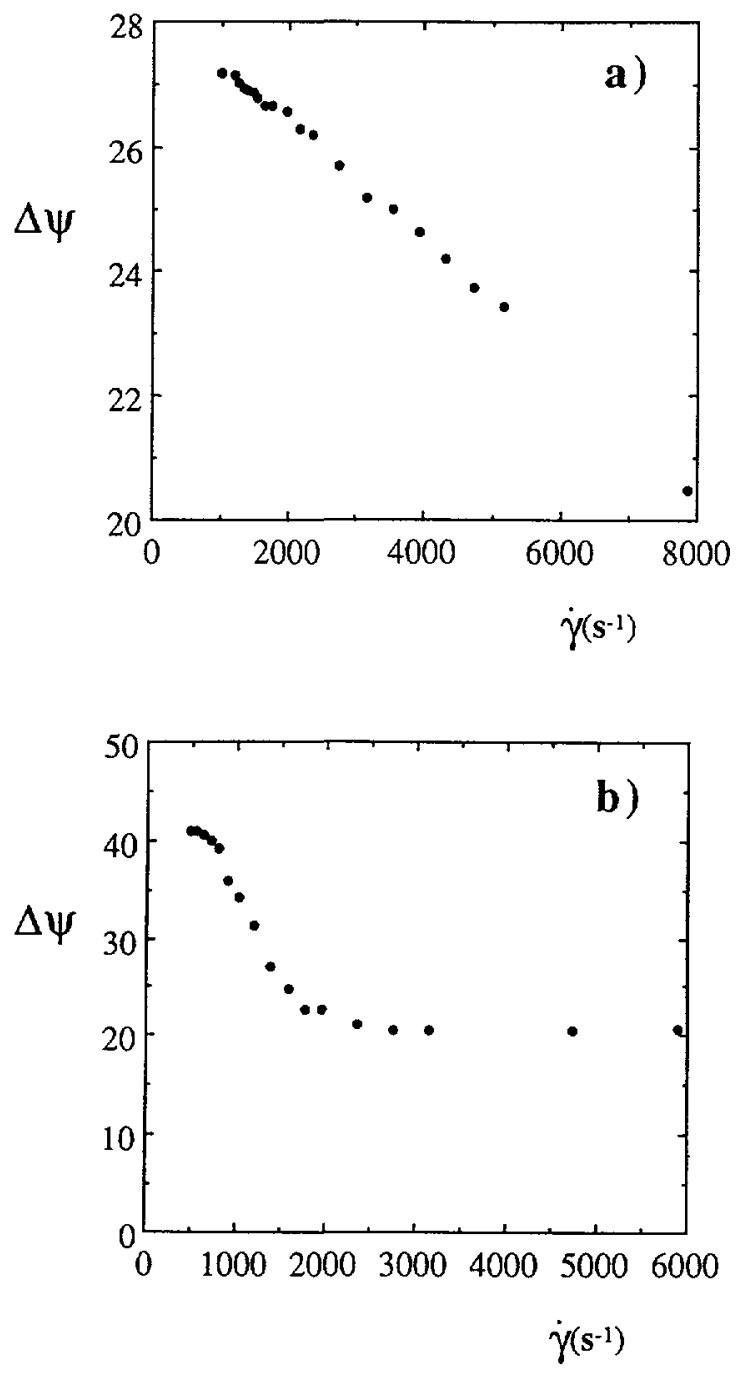

Fig. 28. - Evolution of the mosaic angle for very dilute samples (membrane weight fraction $13 \%$ in a) and $10 \%$ in b)) as a function of the shear rate.

complex behaviour will be probably quite complicated. We list below some ideas along which both theoretical and experimental investigations could be pursued.

We begin with a very interesting work by $\mathrm{P}$. Oswald and $\mathrm{M}$. Kleman related to the flow behaviour of smectic A phases [17, 18]. A key remark made by the above authors is the fact that, whatever the quality of a shear cell, there is no way to control the distance between plates to a precision better than a few $\mu \mathrm{m}$ (in our case the precision is of the order of a few $100 \mu \mathrm{m}$ ). Taking into account that the characteristic distance between smectic layers is of the order of a few $100 \AA$, it is clear that even a perfectly oriented sample will be full of dislocations in order to fill up the space (see Fig. 29). It is natural to think that the natural orientation of a lyotropic smectic A phase between two plates is homeotropic, i.e. with the membranes parallel to the plates. When the plates move, two regimes may be defined : either the dislocations can follow the flow, in what is called a plastic flow regime [17], or they cannot and dramatic events 


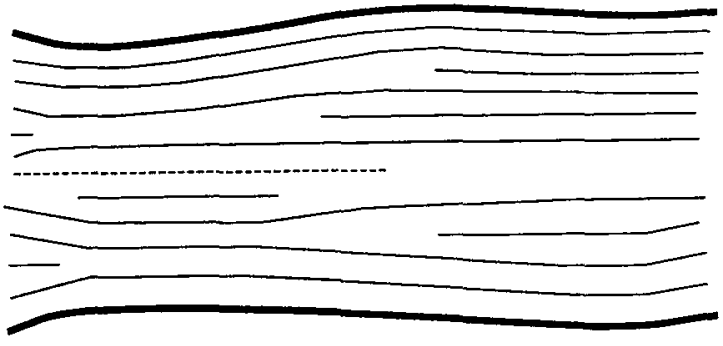

Fig. 29. - Schematic description of the orientation of the membranes between the walls in state I. Dislocations allow to match the fluctuations of the thickness. The dashed line indicates that there are many more layers in the real system that what is shown here (the scale is not preserved).

related to the undulation instability may happen [17-18]. The critical velocity above which the dislocations are unable to follow the flow is related to the permeation coefficient $\lambda_{p}$ and elastic constants of the smectic phase [18].

We have checked that the critical value of the shear rate at which the transition I $\rightarrow$ II appears decreases when $d$ increases (see Fig. 4). When the dislocations can no longer follow the flow, there is a constraint which appears normally to the flow leading to an undulation instability. In the Couette cell this instability develops a rectangular lattice in the X$Z$ plane. When the dilation is large the undulations fuse into a lattice of focal conics [19] and it is then probable that the system cannot flow as a liquid any more. We may think that at this shear rate the system has to find another way of orienting the lamellae to support the flow. From the experimental results we obtained, it seems that a possible solution is to form spherulites which can roll on each other.

The spherulite size can be calculated using a very simple estimate that is supported by more detailed models [20], following Taylor's explanation of the size of emulsions prepared under shear [21]. Considering a spherulite of size $R$ (made of $n=R / d$ layers), we balance the viscous stress :

$$
S_{\mathrm{v}}=\eta R^{2} \dot{\gamma}
$$

with the elastic stress stored in this spherical particle :

$$
S_{\mathrm{e}}=4 \pi(2 \kappa+\bar{\kappa}) / d .
$$

The limiting size $R$ reached is consequently :

$$
R=\sqrt{\frac{4 \pi(2 \kappa+\bar{\kappa})}{\eta d \dot{\gamma}}}
$$

At this level, this has to be considered as a dimensional argument, however more microscopic models of the breaking of a smectic droplet under shear leads to similar expressions [20]. We have already observed that the $\dot{\gamma}^{-1 / 2}$ behaviour has been experimentally verified. One of the problems with this expression is the exact value of the viscosity $\eta$ that has to be put in the formula. This viscosity represents a kind of mean field viscosity felt by the particle in the presence of the neighbouring particles. We measured it dynamically [22] and found that it is of the order of 50 times the viscosity of the solvent and a weak function of the dilution. If the behaviour with the shear rate is well understood by this simple argument, the behaviour with the dilution is not so easily explained. Coming back to figure 11, we may get 
the dependence of the size of the spherulites with the smectic spacing $d$, from the slope of the $D\left(\dot{\gamma}^{-1 / 2}\right.$ ) data. It varies as $1 / d^{2}$ (see Fig. 30), which is much faster than the $d^{-1 / 2}$ expected from equation (7).

We have seen that above a critical shear rate the spherulites are destroyed and a discontinuous transition to a rather well oriented state is observed. Very little is known about the origin of this transition. We may just wonder what is happening to the dislocations (they obviously cannot follow the flow), since we find again a state corresponding to the membranes parallel to the walls. We propose two possible explanations : either there is a « dead layer » where all the defects are located and which is not moving with the flow but sticks on the walls (see Fig. 31a) or the dislocations have no time to form and the system pays the price in compression/dilation energy instead of undulating (see Fig. 31b).
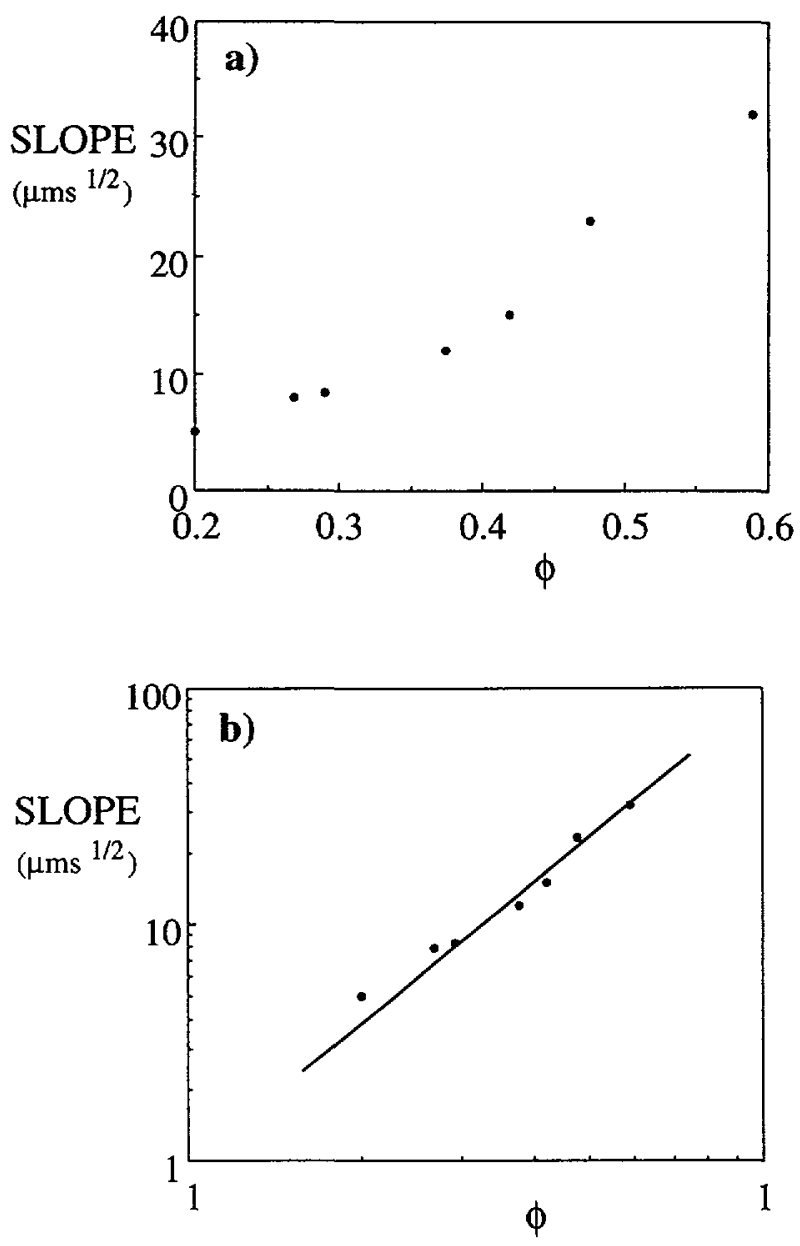

Fig. 30. - Evolution of the slope of the characteristic size in region II as a function of the membrane weight fraction (see Fig. 11), linear scale (a) and logarithmic scale (b). 


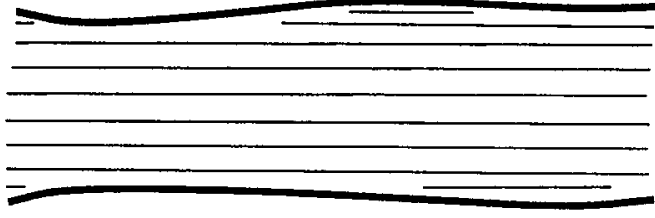

a

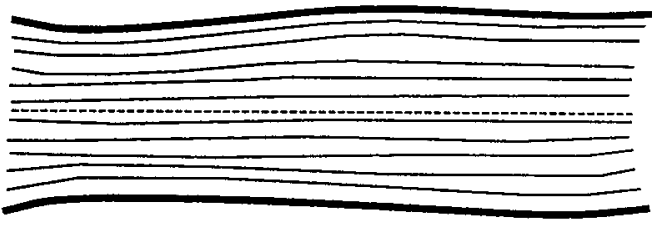

b

Fig. 31. - Schematic representation of the orientation of the membranes in state III, along the flow direction. Two cases are described, either the dislocations are stuck against the walls to match the thickness variation or there is no dislocations in the flow direction and the variation of thickness is compensated by compression and dilation of the lamellar phase.

\section{Acknowledgments.}

The neutron scattering experiments have been performed on the PAXY line of the laboratoire Léon-Brillouin, CEA/CNRS, Saclay, France; we thank L. Noirez and J.-P. Cotton for their kind assistance.

We would like to acknowledge especially C. R. Safinya for discussions and his contribution to our initial interest in the effect of shear on lyotropic systems. We also acknowledge many discussions with M. E. Cates, S. T. Milner, J. Prost and S. Ramaswamy. We thank John Katsaras for a critical reading of the manuscript.

\section{Appendix.}

To determine the orientation of the lamellar phase in region III where there is no further evolution of the transmitted intensity (except for the most dilute samples), we assume, from the neutron scattering analysis that the orientation state is a superposition of all orientations between $\mathrm{A}$ and $\mathrm{C}$. It remains for the orientation distribution to be determined.

The sample under shear is splitted into a sum of uniaxial systems with the same birefringence $\Delta n$ but with optical axes distributed in the $\mathrm{Y}-\mathrm{Z}$ plane. If we add the amplitudes of the polarized plane wave travelling along $\mathbf{k}$ through differently oriented domains the final phase is $\gamma$ :

$$
\gamma=\frac{\pi}{\lambda} \Delta n e \int \rho(\beta) \sin ^{2}(\beta) \mathrm{d} \beta
$$

where $\rho(\beta)$ is the angular distribution of $\mathbf{n}$ with respect to $\mathbf{k}$. 
If we consider a Gaussian distribution, centered when $\mathbf{n}$ is along the $\mathrm{Y}$-axis in order to model a predominantly $\mathrm{C}$ orientation, we have :

$$
\rho(\beta)=\frac{1}{\sqrt{2 \pi} \Delta \psi} \exp \left(-\frac{(\beta-\alpha)^{2}}{2 \Delta \psi^{2}}\right)
$$

for light propagating at an angle $\alpha$ from the $\mathrm{Y}$-axis, where we assume a small $\Delta \psi$ for the distribution normalization. The phase is then given by :

$$
\gamma=\frac{\pi}{2 \lambda} \Delta n \frac{e}{\cos \alpha} \int \frac{1}{\sqrt{2 \pi} \Delta \psi} \exp \left(-\frac{(\beta-\alpha)^{2}}{2 \Delta \psi^{2}}\right)(1-\cos 2 \beta) \mathrm{d} \beta
$$

(recall that the optical path is $e / \cos \alpha$ ), i.e.

$$
\gamma=\frac{\pi}{2 \lambda} \Delta n \frac{e}{\cos \alpha}\left(1-\cos 2 \alpha \mathrm{e}^{-2 \Delta \psi^{2}}\right) .
$$

Since $\Delta \psi$ and $\alpha$ are small, we finally get the relative transmitted intensity :

$$
\frac{I}{I_{0}}=\sin ^{2}\left[\frac{\pi \Delta n e}{\lambda}\left\{\Delta \psi^{2}+\alpha^{2}\left(1-\frac{3 \Delta \psi^{2}}{2}\right)\right\}\right] \text {. }
$$

\section{References}

[1] Ekwall P., Advances in Liquid Crystals, G. M. Brown Ed. (Academic Press, N. Y., 1975).

[2] a) Roux D., Bellocq A.-M., Physics of Amphiphiles, V. Degiorgio, M. Corti Eds. (North Holland, Amsterdam, 1985) ;

b) Bellocq A.-M., Roux D., Microemulsions : Structure and Dynamics, S. Friberg, P. Bothorel Eds. (CRC Press, Boca Baton, 1987) p. 33.

[3] Safinya C. R., Roux D., Smith G. S., Sinha S. K., Dimon P., Clark N. A., Bellocq A.-M., Phys. Rev. Lett. 57 (1986) 2718.

[4] Bassereau P., Marignan J., Porte G., J. Phys. France 48 (1987) 673.

[5] Roux D., Safinya C., J. Phys. France 49 (1988) 307 ;

Richetti P., Kékicheff P., Parker J. L., Ninham B. W., Nature 346 (1990) 252;

Zemb Th., Belloni L., Dubois M., Marcelja S., Prog. Colloid Polym. Sci. 89 (1992) 33.

[6] Strey R., Schoemaecker R., Roux D., Nallet F., Olsson U., J. Chem. Soc. Faraday Trans. 86 (1990) 2253.

[7] Nallet F., Roux D., Prost J., Phys. Rev. Lett. 62 (1989) 276.

Nallet F., Roux D., Prost J., J. Phys. France 50 (1989) 3147.

[8] Nallet F., Roux D., Milner S. T., J. Phys. France 51 (1990) 2333.

[9] Ackerson B. J., Clark N., Phys. Rev. A 30 (1984) 906.

[10] Pieranski P., Contemp. Phys. 24 (1983) 25.

[11] Beysens D., Gladamassi M., J. Phys. France Lett. 40 (1979) 565.

[12] Safinya C. R., Sirota E. B., Plano R. J., Bruinsma R., J. Phys. C 2 (1991) 365.

[13] Orsay Group on Liquid Crystals, J. Phys. Colloq. France 36 (C1) (1975) 305.

[14] Diat O., Roux D., J. Phys. II France 3 (1993) 9.

[15] Roux D., Diat O., French Patent 9204108.

[16] Sirota E. B., Safinya C. R., Plano R. J., Jeppesen C., Bruinsma R. F., to appear : complex fluids MRS proceedings, vol. 248, E. B. Sirota et al. Eds.

[17] Oswald P., Kléman M., J. Phys. France Lett. 43 (1982) L 411.

[18] Oswald P., Ben-Abraham S. I., J. Phys. France 43 (1982) 1193.

[19] Rosenblatt Ch. S., Pindak R., Clark N. A., Meyer R. B., J. Phys. France 38 (1977) 1105.

[20] Roux D., Prost J., Leibler L., unpublished.

[21] Taylor G. I., Proc. R. Soc. London Ser. A 138 (1932) 41 ; 146 (1934) 501.

[22] Roux D., Cates M., unpublished. 